\title{
ON THE RELIABILITY OF THE ESTIMATED INCAPABILITY INDEX
}

\author{
W. L. PEARN ${ }^{1 *}$ AND G. H. LIN ${ }^{2}$ \\ ${ }^{1}$ Department of Industrial Engineering and Management, National Chiao Tung University, Taiwan, Republic of China \\ ${ }^{2}$ Department of Telecommunication Engineering, National Penghu Institute of Technology, Taiwan, Republic of China
}

\begin{abstract}
SUMMARY
Greenwich and Jahr-Schaffrath (1995) introduced the process incapability index $C_{\mathrm{pp}}=C_{\mathrm{ip}}+C_{\mathrm{ia}}$, which provides an uncontaminated separation between information concerning the process precision $\left(C_{\mathrm{ip}}\right)$ and process accuracy $\left(C_{\mathrm{ia}}\right)$. In this paper, we consider the three indices, and investigate the statistical properties of their natural estimators. For the three indices, we obtain their UMVUEs and MLEs, and compare the reliability of the two estimators based on the relative mean square errors. In addition, we construct $90 \%, 95 \%$, and $99 \%$ upper confidence limits, and the maximum values of $\hat{C}_{\mathrm{pp}}$ for which the process is capable $90 \%, 95 \%$, and $99 \%$ of the time. The results obtained in this paper are useful to the practitioners in choosing good estimators and making reliable decisions on judging process capability. Copyright @ 2001 John Wiley \& Sons, Ltd.
\end{abstract}

KEY WORDS: imprecision index; inaccuracy index; UMVUE; MLE; relative mean square error

\section{INTRODUCTION}

Greenwich and Jahr-Schaffrath [1] introduced the process incapability index $C_{\mathrm{pp}}$ to provide numerical measures on process performance for industrial applications. The index $C_{\mathrm{pp}}$ is a simple transformation of $C_{\mathrm{pm}}^{*}$, a general form of the capability index $C_{\mathrm{pm}}$ considered by Chan et al [2], which provides an uncontaminated separation between information concerning the process precision and the process accuracy. The index $C_{\mathrm{pp}}$ is defined as follows:

$$
C_{\mathrm{pp}}=\frac{1}{C_{\mathrm{pm}}^{* 2}}=\left(\frac{\sigma}{D}\right)^{2}+\left(\frac{\mu-T}{D}\right)^{2}
$$

where $\mu$ is the process mean, $\sigma$ is the process standard deviation, $D=\min \{(U S L-T) / 3,(T-$ $L S L) / 3\}, U S L$ and $L S L$ are the upper and the lower specification limits, and $T$ is the target value. If we define $C_{\text {ip }}=(\sigma / D)^{2}$, and $C_{\text {ia }}=[(\mu-T) / D]^{2}$, then $C_{\mathrm{pp}}$ can be expressed as $C_{\mathrm{pp}}=C_{\mathrm{ip}}+C_{\mathrm{ia}}$. Since $C_{\mathrm{ip}}$ measures the process variation relative to the specification tolerance, it has been referred to as the process imprecision index. On the other hand, $C_{\mathrm{ia}}$ measures the relative process departure and has been

\footnotetext{
*Correspondence to: W. L. Pearn, Department of Industrial Engineering and Management, National Chiao Tung University, 1001 Ta Hsuch Road, Hsinchu, Taiwan 30050, Republic of China.
}

Copyright @ 2001 John Wiley \& Sons, Ltd. referred to as the process inaccuracy index. We note that the mathematical relationships $C_{\mathrm{ip}}=1 /\left(C_{\mathrm{p}}\right)^{2}$, and $C_{\mathrm{ia}}=9\left(1-C_{\mathrm{a}}\right)^{2}$ can be established, where $C_{\mathrm{p}}$ and $C_{\mathrm{a}}$ are two basic process capability indices considered by Kane [3] and Pearn et al. [4].

In this paper, we consider the three indices $C_{\mathrm{ip}}$, $C_{\mathrm{ia}}$, and $C_{\mathrm{pp}}$ and investigate the statistical properties of their natural estimators. For $C_{\mathrm{ip}}$, we show that the natural estimator is the UMVUE, which is consistent and asymptotically efficient. We also obtain the MLE (maximum likelihood estimator), which has smaller mean square error than the UMVUE (uniformly minimum variance unbiased estimator), hence it is more reliable, particularly, for short production run applications. For $C_{\mathrm{ia}}$, we show that the natural estimator is the MLE. We also obtain the UMVUE, which is shown to be more reliable than the MLE for applications with $n \geq 4$. We show that the UMVUE is consistent and asymptotically efficient. For $C_{\mathrm{pp}}$, we show that the natural estimator is the MLE and also the UMVUE, which is consistent and asymptotically efficient. In addition, we construct tables of 90\%, 95\%, and $99 \%$ upper confidence limits for $C_{\mathrm{pp}}$ based on the UMVUE. We also construct tables of the maximum values of $\hat{C}_{\mathrm{pp}}$ under $\mu=T$ for which the process is capable. The estimators we recommend have all 
the desired statistical properties, and are considered reliable.

\section{ESTIMATING PROCESS IMPRECISION}

To estimate the process imprecision, we consider the natural estimator $\hat{C}_{\text {ip }}$ defined as follows, where $S_{n-1}=\left[\sum_{i=1}^{n}\left(X_{i}-\bar{X}\right)^{2} /(n-1)\right]^{1 / 2}$ is the conventional estimator of the process standard deviation $\sigma$,

$$
\hat{C}_{\mathrm{ip}}=\frac{1}{n-1} \sum_{i=1}^{n} \frac{\left(X_{i}-\bar{X}\right)^{2}}{D^{2}}=\frac{S_{n-1}^{2}}{D^{2}}
$$

The natural estimator $\hat{C}_{\text {ip }}$ can be rewritten as

$$
\hat{C}_{\mathrm{ip}}=\frac{C_{\mathrm{ip}}}{n-1} \frac{(n-1) \hat{C}_{\mathrm{ip}}}{C_{\mathrm{ip}}}=\frac{C_{\mathrm{ip}}}{n-1} \sum_{i=1}^{n} \frac{\left(X_{i}-\bar{X}\right)^{2}}{\sigma^{2}}
$$

If the process follows the normal distribution, then $\hat{C}_{\text {ip }}$ is distributed as $\left[C_{\text {ip }} /(n-1)\right] \chi_{n-1}^{2}$, where $\chi_{n-1}^{2}$ is a chi-squared distribution with $(n-1)$ degrees of freedom. The probability density function of $\hat{C}_{\text {ip }}$ can be easily derived as

$$
\begin{aligned}
f(y)= & \left\{\left[(n-1) y /\left(2 C_{\mathrm{ip}}\right)\right]^{(n-1) / 2}\right. \\
& \left.\times \exp \left[-(n-1) y /\left(2 C_{\mathrm{ip}}\right)\right]\right\} \\
& \times\{y \Gamma[(n-1) / 2]\}^{-1}, \quad \text { for } y>0
\end{aligned}
$$

The $r$ th moment, the expected value, the variance, and the mean squared error of $\hat{C}_{\text {ip }}$ can be calculated as follows:

$$
\begin{aligned}
\mathrm{E}\left(\hat{C}_{\mathrm{ip}}\right)^{r} & =\frac{\Gamma\{[(n-1) / 2]+r\}}{\Gamma[(n-1) / 2]}\left(\frac{2 C_{\mathrm{ip}}}{n-1}\right)^{r} \\
\mathrm{E}\left(\hat{C}_{\mathrm{ip}}\right) & =\left(\frac{C_{\mathrm{ip}}}{n-1}\right) \mathrm{E}\left(\chi_{n-1}^{2}\right) \\
& =C_{\mathrm{ip}} \\
\operatorname{Var}\left(\hat{C}_{\mathrm{ip}}\right) & =\left(\frac{C_{\mathrm{ip}}}{n-1}\right)^{2} \operatorname{Var}\left(\chi_{n-1}^{2}\right) \\
& =\left(\frac{C_{\mathrm{ip}}}{n-1}\right)^{2} 2(n-1) \\
& =\frac{2 C_{\mathrm{ip}}^{2}}{n-1} \\
\operatorname{MSE}\left(\hat{C}_{\mathrm{ip}}\right) & =\mathrm{E}\left(\hat{C}_{\mathrm{ip}}-C_{\mathrm{ip}}\right)^{2} \\
& =\operatorname{Var}\left(\hat{C}_{\mathrm{ip}}\right)+\left[\mathrm{E}\left(\hat{C}_{\mathrm{ip}}\right)-C_{\mathrm{ip}}\right]^{2} \\
& =\frac{2 C_{\mathrm{ip}}^{2}}{n-1}
\end{aligned}
$$

If the process characteristic is normally distributed, then we can show that the natural estimator $\hat{C}_{\mathrm{ip}}$ is Copyright @ 2001 John Wiley \& Sons, Ltd. the UMVUE of $C_{\mathrm{ip}}$, which is consistent. We can also show that $\sqrt{n}\left(\hat{C}_{\mathrm{ip}}-C_{\mathrm{ip}}\right)$ converges to $N\left(0,2 C_{\mathrm{ip}}^{2}\right)$ in distribution, and that $\hat{C}_{\mathrm{ip}}$ is asymptotically efficient (see the Appendix for the proofs). Thus, in realworld applications using $\hat{C}_{\mathrm{ip}}$, which has all the desired statistical properties, as an estimate of $C_{\mathrm{ip}}$ would be reasonable.

We note that by multiplying the UMVUE $\hat{C}_{\text {ip }}$ by the constant $c_{n}=(n-1) / n$, we obtain the MLE of $C_{\mathrm{ip}}$. We can show that the MLE $\hat{C}_{\mathrm{ip}}^{\prime}$ is consistent, and is asymptotically unbiased. We can also show that $\sqrt{n}\left(\hat{C}_{\mathrm{ip}}^{\prime}-C_{\mathrm{ip}}\right)$ converges to $N\left(0,2 C_{\mathrm{ip}}^{2}\right)$ in distribution, and that $\hat{C}_{\mathrm{ip}}^{\prime}$ is asymptotically efficient. Since $c_{n}<1$, then $\hat{C}_{\mathrm{ip}}^{\prime}=c_{n} \hat{C}_{\mathrm{ip}}$ underestimates $C_{\mathrm{ip}}$ but with smaller variance. In fact, we may calculate

$$
\operatorname{MSE}\left(\hat{C}_{\mathrm{ip}}^{\prime}\right)=\left[(2 n-1) / n^{2}\right]\left(C_{\mathrm{ip}}\right)^{2}
$$

and obtain

$$
\begin{aligned}
& \operatorname{MSE}\left(\hat{C}_{\mathrm{ip}}\right)-\operatorname{MSE}\left(\hat{C}_{\mathrm{ip}}^{\prime}\right) \\
& \quad=\left[(3 n-1) / n^{2}(n-1)\right]\left(C_{\mathrm{ip}}\right)^{2}>0, \text { for all } n
\end{aligned}
$$

Therefore, the MLE $\hat{C}_{\mathrm{ip}}^{\prime}$ has smaller mean squared error than the UMVUE $\hat{C}_{\mathrm{ip}}$, hence it is more reliable, particularly for short production run applications.

Tables 1(a) and 1(b) display the relative error of the UMVUE $\hat{C}_{\text {ip }}$, defined as $\left[\operatorname{MSE} E_{\mathrm{R}}\left(\hat{C}_{\mathrm{ip}}\right)\right]^{1 / 2}=\left\{\mathrm{E}\left[\left(\hat{C}_{\mathrm{ip}}-\right.\right.\right.$ $\left.\left.\left.C_{\text {ip }}\right) / C_{\text {ip }}\right]^{2}\right\}^{1 / 2}$, for sample sizes $n=2(1) 50$, and $60(10) 550$, and some commonly used values of $C_{\mathrm{ip}}=$ $1.00,0.56,0.44,0.36$, and 0.25 , equivalent to $C_{\mathrm{p}}=$ $1.00,1.33,1.50,1.67$, and 2.00 , covering the range of the precision requirements for most applications.

Precision requirement:

Capable: $0.56 \leq C_{\text {ip }} \leq 1.00$

Satisfactory: $0.44 \leq C_{\text {ip }} \leq 0.56$

Good: $0.36 \leq C_{\text {ip }} \leq 0.44$

Excellent: $0.25 \leq C_{\mathrm{ip}} \leq 0.36$

Super: $C_{\text {ip }} \leq 0.25$

The square root of the relative mean squared error is a direct measurement, which presents the expected relative error of the estimation from the true $C_{\mathrm{ip}}$. We note that for UMVUE $\left.\hat{C}_{\mathrm{ip}}, \operatorname{MSSE}_{\mathrm{R}}\left(\hat{C}_{\mathrm{ip}}\right)\right]^{1 / 2}=$ $[2 /(n-1)]^{1 / 2}$, which is a function of the sample size $n$ only. Therefore, $\left[\operatorname{MSE}_{\mathrm{R}}\left(\hat{C}_{\mathrm{ip}}\right)\right]^{1 / 2}$ values are the same for all $C_{\text {ip }}$ values. For example, with $n=300$ we have $\left[\operatorname{MSE} E_{\mathrm{R}}\left(\hat{C}_{\mathrm{ip}}\right)\right]^{1 / 2}=0.0818$. Thus, for $n=300$, we expect that the average error of $\hat{C}_{\text {ip }}$ would be no greater than $8.18 \%$ of the true $C_{\mathrm{p}}$. Tables 2(a) and 2(b) display the relative error, $\left[\operatorname{MSE}_{\mathrm{R}}\left(\hat{C}_{\mathrm{ip}}^{\prime}\right)\right]^{1 / 2}$, of the MLE $\hat{C}_{\text {ip }}^{\prime}$. We note that $\left[\operatorname{MSE}_{\mathrm{R}}\left(\hat{C}_{\mathrm{ip}}^{\prime}\right)\right]^{1 / 2}=\left[(2 n-1) / n^{2}\right]^{1 / 2}$,

Qual. Reliab. Engng. Int. 2001; 17: 279-290 
Table 1. $\left[\operatorname{MSE}_{\mathrm{R}}\left(\hat{C}_{\mathrm{ip}}\right)\right]^{1 / 2}$ for various $C_{\mathrm{ip}}$, and sample sizes (a) $n=2(1) 50$ and (b) $n=60(10) 550$

\begin{tabular}{|c|c|c|c|c|c|c|c|c|c|c|c|}
\hline \multirow[b]{2}{*}{$n$} & \multicolumn{5}{|c|}{$C_{\mathrm{ip}}$} & \multirow[b]{2}{*}{$n$} & \multicolumn{5}{|c|}{$C_{\mathrm{ip}}$} \\
\hline & 1.00 & 0.56 & 0.44 & 0.36 & 0.25 & & 1.00 & 0.56 & 0.44 & 0.36 & 0.25 \\
\hline 1 & $* * * * * *$ & $* * * * * *$ & $* * * * * *$ & $* * * * * *$ & $* * * * * *$ & 60 & 0.1841 & 0.1841 & 0.1841 & 0.1841 & 0.1841 \\
\hline 2 & 1.4142 & 1.4142 & 1.4142 & 1.4142 & 1.4142 & 70 & 0.1703 & 0.1703 & 0.1703 & 0.1703 & 0.1703 \\
\hline 3 & 1.0000 & 1.0000 & 1.0000 & 1.0000 & 1.0000 & 80 & 0.1591 & 0.1591 & 0.1591 & 0.1591 & 0.1591 \\
\hline 4 & 0.8165 & 0.8165 & 0.8165 & 0.8165 & 0.8165 & 90 & 0.1499 & 0.1499 & 0.1499 & 0.1499 & 0.1499 \\
\hline 5 & 0.7071 & 0.7071 & 0.7071 & 0.7071 & 0.7071 & 100 & 0.1421 & 0.1421 & 0.1421 & 0.1421 & 0.1421 \\
\hline 6 & 0.6325 & 0.6325 & 0.6325 & 0.6325 & 0.6325 & 110 & 0.1355 & 0.1355 & 0.1355 & 0.1355 & 0.1355 \\
\hline 7 & 0.5774 & 0.5774 & 0.5774 & 0.5774 & 0.5774 & 120 & 0.1296 & 0.1296 & 0.1296 & 0.1296 & 0.1296 \\
\hline 8 & 0.5345 & 0.5345 & 0.5345 & 0.5345 & 0.5345 & 130 & 0.1245 & 0.1245 & 0.1245 & 0.1245 & 0.1245 \\
\hline 9 & 0.5000 & 0.5000 & 0.5000 & 0.5000 & 0.5000 & 140 & 0.1200 & 0.1200 & 0.1200 & 0.1200 & 0.1200 \\
\hline 10 & 0.4714 & 0.4714 & 0.4714 & 0.4714 & 0.4714 & 150 & 0.1159 & 0.1159 & 0.1159 & 0.1159 & 0.1159 \\
\hline 11 & 0.4472 & 0.4472 & 0.4472 & 0.4472 & 0.4472 & 160 & 0.1122 & 0.1122 & 0.1122 & 0.1122 & 0.1122 \\
\hline 12 & 0.4264 & 0.4264 & 0.4264 & 0.4264 & 0.4264 & 170 & 0.1088 & 0.1088 & 0.1088 & 0.1088 & 0.1088 \\
\hline 13 & 0.4082 & 0.4082 & 0.4082 & 0.4082 & 0.4082 & 180 & 0.1057 & 0.1057 & 0.1057 & 0.1057 & 0.1057 \\
\hline 14 & 0.3922 & 0.3922 & 0.3922 & 0.3922 & 0.3922 & 190 & 0.1029 & 0.1029 & 0.1029 & 0.1029 & 0.1029 \\
\hline 15 & 0.3780 & 0.3780 & 0.3780 & 0.3780 & 0.3780 & 200 & 0.1003 & 0.1003 & 0.1003 & 0.1003 & 0.1003 \\
\hline 16 & 0.3651 & 0.3651 & 0.3651 & 0.3651 & 0.3651 & 210 & 0.0978 & 0.0978 & 0.0978 & 0.0978 & 0.0978 \\
\hline 17 & 0.3536 & 0.3536 & 0.3536 & 0.3536 & 0.3536 & 220 & 0.0956 & 0.0956 & 0.0956 & 0.0956 & 0.0956 \\
\hline 18 & 0.3430 & 0.3430 & 0.3430 & 0.3430 & 0.3430 & 230 & 0.0935 & 0.0935 & 0.0935 & 0.0935 & 0.0935 \\
\hline 19 & 0.3333 & 0.3333 & 0.3333 & 0.3333 & 0.3333 & 240 & 0.0915 & 0.0915 & 0.0915 & 0.0915 & 0.0915 \\
\hline 20 & 0.3244 & 0.3244 & 0.3244 & 0.3244 & 0.3244 & 250 & 0.0896 & 0.0896 & 0.0896 & 0.0896 & 0.0896 \\
\hline 21 & 0.3162 & 0.3162 & 0.3162 & 0.3162 & 0.3162 & 260 & 0.0879 & 0.0879 & 0.0879 & 0.0879 & 0.0879 \\
\hline 22 & 0.3086 & 0.3086 & 0.3086 & 0.3086 & 0.3086 & 270 & 0.0862 & 0.0862 & 0.0862 & 0.0862 & 0.0862 \\
\hline 23 & 0.3015 & 0.3015 & 0.3015 & 0.3015 & 0.3015 & 280 & 0.0847 & 0.0847 & 0.0847 & 0.0847 & 0.0847 \\
\hline 24 & 0.2949 & 0.2949 & 0.2949 & 0.2949 & 0.2949 & 290 & 0.0832 & 0.0832 & 0.0832 & 0.0832 & 0.0832 \\
\hline 25 & 0.2887 & 0.2887 & 0.2887 & 0.2887 & 0.2887 & 300 & 0.0818 & 0.0818 & 0.0818 & 0.0818 & 0.0818 \\
\hline 26 & 0.2828 & 0.2828 & 0.2828 & 0.2828 & 0.2828 & 310 & 0.0805 & 0.0805 & 0.0805 & 0.0805 & 0.0805 \\
\hline 27 & 0.2774 & 0.2774 & 0.2774 & 0.2774 & 0.2774 & 320 & 0.0792 & 0.0792 & 0.0792 & 0.0792 & 0.0792 \\
\hline 28 & 0.2722 & 0.2722 & 0.2722 & 0.2722 & 0.2722 & 330 & 0.0780 & 0.0780 & 0.0780 & 0.0780 & 0.0780 \\
\hline 29 & 0.2673 & 0.2673 & 0.2673 & 0.2673 & 0.2673 & 340 & 0.0768 & 0.0768 & 0.0768 & 0.0768 & 0.0768 \\
\hline 30 & 0.2626 & 0.2626 & 0.2626 & 0.2626 & 0.2626 & 350 & 0.0757 & 0.0757 & 0.0757 & 0.0757 & 0.0757 \\
\hline 31 & 0.2582 & 0.2582 & 0.2582 & 0.2582 & 0.2582 & 360 & 0.0746 & 0.0746 & 0.0746 & 0.0746 & 0.0746 \\
\hline 32 & 0.2540 & 0.2540 & 0.2540 & 0.2540 & 0.2540 & 370 & 0.0736 & 0.0736 & 0.0736 & 0.0736 & 0.0736 \\
\hline 33 & 0.2500 & 0.2500 & 0.2500 & 0.2500 & 0.2500 & 380 & 0.0726 & 0.0726 & 0.0726 & 0.0726 & 0.0726 \\
\hline 34 & 0.2462 & 0.2462 & 0.2462 & 0.2462 & 0.2462 & 390 & 0.0717 & 0.0717 & 0.0717 & 0.0717 & 0.0717 \\
\hline 35 & 0.2425 & 0.2425 & 0.2425 & 0.2425 & 0.2425 & 400 & 0.0708 & 0.0708 & 0.0708 & 0.0708 & 0.0708 \\
\hline 36 & 0.2390 & 0.2390 & 0.2390 & 0.2390 & 0.2390 & 410 & 0.0699 & 0.0699 & 0.0699 & 0.0699 & 0.0699 \\
\hline 37 & 0.2357 & 0.2357 & 0.2357 & 0.2357 & 0.2357 & 420 & 0.0691 & 0.0691 & 0.0691 & 0.0691 & 0.0691 \\
\hline 38 & 0.2325 & 0.2325 & 0.2325 & 0.2325 & 0.2325 & 430 & 0.0683 & 0.0683 & 0.0683 & 0.0683 & 0.0683 \\
\hline 39 & 0.2294 & 0.2294 & 0.2294 & 0.2294 & 0.2294 & 440 & 0.0675 & 0.0675 & 0.0675 & 0.0675 & 0.0675 \\
\hline 40 & 0.2265 & 0.2265 & 0.2265 & 0.2265 & 0.2265 & 450 & 0.0667 & 0.0667 & 0.0667 & 0.0667 & 0.0667 \\
\hline 41 & 0.2236 & 0.2236 & 0.2236 & 0.2236 & 0.2236 & 460 & 0.0660 & 0.0660 & 0.0660 & 0.0660 & 0.0660 \\
\hline 42 & 0.2209 & 0.2209 & 0.2209 & 0.2209 & 0.2209 & 470 & 0.0653 & 0.0653 & 0.0653 & 0.0653 & 0.0653 \\
\hline 43 & 0.2182 & 0.2182 & 0.2182 & 0.2182 & 0.2182 & 480 & 0.0646 & 0.0646 & 0.0646 & 0.0646 & 0.0646 \\
\hline 44 & 0.2157 & 0.2157 & 0.2157 & 0.2157 & 0.2157 & 490 & 0.0640 & 0.0640 & 0.0640 & 0.0640 & 0.0640 \\
\hline 45 & 0.2132 & 0.2132 & 0.2132 & 0.2132 & 0.2132 & 500 & 0.0633 & 0.0633 & 0.0633 & 0.0633 & 0.0633 \\
\hline 46 & 0.2108 & 0.2108 & 0.2108 & 0.2108 & 0.2108 & 510 & 0.0627 & 0.0627 & 0.0627 & 0.0627 & 0.0627 \\
\hline 47 & 0.2085 & 0.2085 & 0.2085 & 0.2085 & 0.2085 & 520 & 0.0621 & 0.0621 & 0.0621 & 0.0621 & 0.0621 \\
\hline 48 & 0.2063 & 0.2063 & 0.2063 & 0.2063 & 0.2063 & 530 & 0.0615 & 0.0615 & 0.0615 & 0.0615 & 0.0615 \\
\hline 49 & 0.2041 & 0.2041 & 0.2041 & 0.2041 & 0.2041 & 540 & 0.0609 & 0.0609 & 0.0609 & 0.0609 & 0.0609 \\
\hline 50 & 0.2020 & 0.2020 & 0.2020 & 0.2020 & 0.2020 & 550 & 0.0604 & 0.0604 & 0.0604 & 0.0604 & 0.0604 \\
\hline
\end{tabular}


Table 2. $\left[\operatorname{MSE}_{\mathrm{R}}\left(\hat{C}_{\mathrm{ip}}^{\prime}\right)\right]^{1 / 2}$ for various $C_{\mathrm{ip}}$, and sample sizes (a) $n=1(1) 50$ and (b) $n=60(10) 550$

\begin{tabular}{|c|c|c|c|c|c|c|c|c|c|c|c|}
\hline \multirow[b]{2}{*}{$n$} & \multicolumn{5}{|c|}{$C_{\text {ip }}$} & \multirow[b]{2}{*}{$n$} & \multicolumn{5}{|c|}{$C_{\mathrm{ip}}$} \\
\hline & 1.00 & 0.56 & 0.44 & 0.36 & 0.25 & & 1.00 & 0.56 & 0.44 & 0.36 & 0.25 \\
\hline 1 & 1.0000 & 1.0000 & 1.0000 & 1.0000 & 1.0000 & 60 & 0.1818 & 0.1818 & 0.1818 & 0.1818 & 0.1818 \\
\hline 2 & 0.8660 & 0.8660 & 0.8660 & 0.8660 & 0.8660 & 70 & 0.1684 & 0.1684 & 0.1684 & 0.1684 & 0.1684 \\
\hline 3 & 0.7454 & 0.7454 & 0.7454 & 0.7454 & 0.7454 & 80 & 0.1576 & 0.1576 & 0.1576 & 0.1576 & 0.1576 \\
\hline 4 & 0.6614 & 0.6614 & 0.6614 & 0.6614 & 0.6614 & 90 & 0.1487 & 0.1487 & 0.1487 & 0.1487 & 0.1487 \\
\hline 5 & 0.6000 & 0.6000 & 0.6000 & 0.6000 & 0.6000 & 100 & 0.1411 & 0.1411 & 0.1411 & 0.1411 & 0.1411 \\
\hline 6 & 0.5528 & 0.5528 & 0.5528 & 0.5528 & 0.5528 & 110 & 0.1345 & 0.1345 & 0.1345 & 0.1345 & 0.1345 \\
\hline 7 & 0.5151 & 0.5151 & 0.5151 & 0.5151 & 0.5151 & 120 & 0.1288 & 0.1288 & 0.1288 & 0.1288 & 0.1288 \\
\hline 8 & 0.4841 & 0.4841 & 0.4841 & 0.4841 & 0.4841 & 130 & 0.1238 & 0.1238 & 0.1238 & 0.1238 & 0.1238 \\
\hline 9 & 0.4581 & 0.4581 & 0.4581 & 0.4581 & 0.4581 & 140 & 0.1193 & 0.1193 & 0.1193 & 0.1193 & 0.1193 \\
\hline 10 & 0.4359 & 0.4359 & 0.4359 & 0.4359 & 0.4359 & 150 & 0.1153 & 0.1153 & 0.1153 & 0.1153 & 0.1153 \\
\hline 11 & 0.4166 & 0.4166 & 0.4166 & 0.4166 & 0.4166 & 160 & 0.1116 & 0.1116 & 0.1116 & 0.1116 & 0.1116 \\
\hline 12 & 0.3997 & 0.3997 & 0.3997 & 0.3997 & 0.3997 & 170 & 0.1083 & 0.1083 & 0.1083 & 0.1083 & 0.1083 \\
\hline 13 & 0.3846 & 0.3846 & 0.3846 & 0.3846 & 0.3846 & 180 & 0.1053 & 0.1053 & 0.1053 & 0.1053 & 0.1053 \\
\hline 14 & 0.3712 & 0.3712 & 0.3712 & 0.3712 & 0.3712 & 190 & 0.1025 & 0.1025 & 0.1025 & 0.1025 & 0.1025 \\
\hline 15 & 0.3590 & 0.3590 & 0.3590 & 0.3590 & 0.3590 & 200 & 0.0999 & 0.0999 & 0.0999 & 0.0999 & 0.0999 \\
\hline 16 & 0.3480 & 0.3480 & 0.3480 & 0.3480 & 0.3480 & 210 & 0.0975 & 0.0975 & 0.0975 & 0.0975 & 0.0975 \\
\hline 17 & 0.3379 & 0.3379 & 0.3379 & 0.3379 & 0.3379 & 220 & 0.0952 & 0.0952 & 0.0952 & 0.0952 & 0.0952 \\
\hline 18 & 0.3287 & 0.3287 & 0.3287 & 0.3287 & 0.3287 & 230 & 0.0931 & 0.0931 & 0.0931 & 0.0931 & 0.0931 \\
\hline 19 & 0.3201 & 0.3201 & 0.3201 & 0.3201 & 0.3201 & 240 & 0.0912 & 0.0912 & 0.0912 & 0.0912 & 0.0912 \\
\hline 20 & 0.3123 & 0.3123 & 0.3123 & 0.3123 & 0.3123 & 250 & 0.0894 & 0.0894 & 0.0894 & 0.0894 & 0.0894 \\
\hline 21 & 0.3049 & 0.3049 & 0.3049 & 0.3049 & 0.3049 & 260 & 0.0876 & 0.0876 & 0.0876 & 0.0876 & 0.0876 \\
\hline 22 & 0.2981 & 0.2981 & 0.2981 & 0.2981 & 0.2981 & 270 & 0.0860 & 0.0860 & 0.0860 & 0.0860 & 0.0860 \\
\hline 23 & 0.2917 & 0.2917 & 0.2917 & 0.2917 & 0.2917 & 280 & 0.0844 & 0.0844 & 0.0844 & 0.0844 & 0.0844 \\
\hline 24 & 0.2857 & 0.2857 & 0.2857 & 0.2857 & 0.2857 & 290 & 0.0830 & 0.0830 & 0.0830 & 0.0830 & 0.0830 \\
\hline 25 & 0.2800 & 0.2800 & 0.2800 & 0.2800 & 0.2800 & 300 & 0.0816 & 0.0816 & 0.0816 & 0.0816 & 0.0816 \\
\hline 26 & 0.2747 & 0.2747 & 0.2747 & 0.2747 & 0.2747 & 310 & 0.0803 & 0.0803 & 0.0803 & 0.0803 & 0.0803 \\
\hline 27 & 0.2696 & 0.2696 & 0.2696 & 0.2696 & 0.2696 & 320 & 0.0790 & 0.0790 & 0.0790 & 0.0790 & 0.0790 \\
\hline 28 & 0.2649 & 0.2649 & 0.2649 & 0.2649 & 0.2649 & 330 & 0.0778 & 0.0778 & 0.0778 & 0.0778 & 0.0778 \\
\hline 29 & 0.2603 & 0.2603 & 0.2603 & 0.2603 & 0.2603 & 340 & 0.0766 & 0.0766 & 0.0766 & 0.0766 & 0.0766 \\
\hline 30 & 0.2560 & 0.2560 & 0.2560 & 0.2560 & 0.2560 & 350 & 0.0755 & 0.0755 & 0.0755 & 0.0755 & 0.0755 \\
\hline 31 & 0.2519 & 0.2519 & 0.2519 & 0.2519 & 0.2519 & 360 & 0.0745 & 0.0745 & 0.0745 & 0.0745 & 0.0745 \\
\hline 32 & 0.2480 & 0.2480 & 0.2480 & 0.2480 & 0.2480 & 370 & 0.0735 & 0.0735 & 0.0735 & 0.0735 & 0.0735 \\
\hline 33 & 0.2443 & 0.2443 & 0.2443 & 0.2443 & 0.2443 & 380 & 0.0725 & 0.0725 & 0.0725 & 0.0725 & 0.0725 \\
\hline 34 & 0.2407 & 0.2407 & 0.2407 & 0.2407 & 0.2407 & 390 & 0.0716 & 0.0716 & 0.0716 & 0.0716 & 0.0716 \\
\hline 35 & 0.2373 & 0.2373 & 0.2373 & 0.2373 & 0.2373 & 400 & 0.0707 & 0.0707 & 0.0707 & 0.0707 & 0.0707 \\
\hline 36 & 0.2341 & 0.2341 & 0.2341 & 0.2341 & 0.2341 & 410 & 0.0698 & 0.0698 & 0.0698 & 0.0698 & 0.0698 \\
\hline 37 & 0.2309 & 0.2309 & 0.2309 & 0.2309 & 0.2309 & 420 & 0.0690 & 0.0690 & 0.0690 & 0.0690 & 0.0690 \\
\hline 38 & 0.2279 & 0.2279 & 0.2279 & 0.2279 & 0.2279 & 430 & 0.0682 & 0.0682 & 0.0682 & 0.0682 & 0.0682 \\
\hline 39 & 0.2250 & 0.2250 & 0.2250 & 0.2250 & 0.2250 & 440 & 0.0674 & 0.0674 & 0.0674 & 0.0674 & 0.0674 \\
\hline 40 & 0.2222 & 0.2222 & 0.2222 & 0.2222 & 0.2222 & 450 & 0.0666 & 0.0666 & 0.0666 & 0.0666 & 0.0666 \\
\hline 41 & 0.2195 & 0.2195 & 0.2195 & 0.2195 & 0.2195 & 460 & 0.0659 & 0.0659 & 0.0659 & 0.0659 & 0.0659 \\
\hline 42 & 0.2169 & 0.2169 & 0.2169 & 0.2169 & 0.2169 & 470 & 0.0652 & 0.0652 & 0.0652 & 0.0652 & 0.0652 \\
\hline 43 & 0.2144 & 0.2144 & 0.2144 & 0.2144 & 0.2144 & 480 & 0.0645 & 0.0645 & 0.0645 & 0.0645 & 0.0645 \\
\hline 44 & 0.2120 & 0.2120 & 0.2120 & 0.2120 & 0.2120 & 490 & 0.0639 & 0.0639 & 0.0639 & 0.0639 & 0.0639 \\
\hline 45 & 0.2096 & 0.2096 & 0.2096 & 0.2096 & 0.2096 & 500 & 0.0632 & 0.0632 & 0.0632 & 0.0632 & 0.0632 \\
\hline 46 & 0.2074 & 0.2074 & 0.2074 & 0.2074 & 0.2074 & 510 & 0.0626 & 0.0626 & 0.0626 & 0.0626 & 0.0626 \\
\hline 47 & 0.2052 & 0.2052 & 0.2052 & 0.2052 & 0.2052 & 520 & 0.0620 & 0.0620 & 0.0620 & 0.0620 & 0.0620 \\
\hline 48 & 0.2031 & 0.2031 & 0.2031 & 0.2031 & 0.2031 & 530 & 0.0614 & 0.0614 & 0.0614 & 0.0614 & 0.0614 \\
\hline 49 & 0.2010 & 0.2010 & 0.2010 & 0.2010 & 0.2010 & 540 & 0.0608 & 0.0608 & 0.0608 & 0.0608 & 0.0608 \\
\hline 50 & 0.1990 & 0.1990 & 0.1990 & 0.1990 & 0.1990 & 550 & 0.0603 & 0.0603 & 0.0603 & 0.0603 & 0.0603 \\
\hline
\end{tabular}


which is also a function of the sample size $n$ only. Thus, $\left[\operatorname{MSE} E_{\mathrm{R}}\left(\hat{C}_{\mathrm{ip}}^{\prime}\right)\right]^{1 / 2}$ values are the same for all $C_{\mathrm{ip}}$ values.

For short run applications (such as accepting a supplier providing short production runs in QS-9000 certification), the difference between the two relative errors is considered significant for sample sizes $n \leq$ 35 , and we strongly recommend using the MLE $\hat{C}_{\mathrm{ip}}^{\prime}$ rather than the UMVUE $\hat{C}_{\mathrm{ip}}$. For other applications with sample sizes $n>35$, the difference between the two estimators is negligible (less than $0.52 \%$ ).

\section{ESTIMATING PROCESS INACCURACY}

To estimate the process inaccuracy, we consider the natural estimator $\hat{C}_{\text {ia }}$ defined as the following, where $\bar{X}=\sum_{i=1}^{n} X_{i} / n$ is the conventional estimator of the process mean $\mu$. We note that the estimator $\hat{C}_{\text {ia }}$ can also be written as a function of $C_{\mathrm{in}}$ :

$$
\hat{C}_{\mathrm{ia}}=\frac{(\bar{X}-T)^{2}}{D^{2}}=\frac{C_{\mathrm{ip}}}{n} \frac{n \hat{C}_{\mathrm{ia}}}{C_{\mathrm{ip}}}=\frac{C_{\mathrm{ip}}}{n} \frac{n(\bar{X}-T)^{2}}{\sigma^{2}}
$$

If the process characteristic is normally distributed, then the estimator $\hat{C}_{\mathrm{ia}}$ is distributed as $\left[C_{\mathrm{ip}} / n\right] \chi_{1}^{2}(\delta)$, where $\chi_{1}^{2}(\delta)$ is a non-central chi-squared distribution with one degree of freedom and non-centrality parameter $\delta=n(\mu-T)^{2} / \sigma^{2}$. Therefore, the probability density function of $\hat{C}_{\text {ia }}$ can be expressed as

$$
\begin{aligned}
g(y)= & \sum_{k=0}^{\infty}\left\{\frac{\left[(n y) /\left(2 C_{\mathrm{ip}}\right)\right]^{k+\frac{1}{2}} \exp \left[-(n y) /\left(2 C_{\mathrm{ip}}\right)\right]}{y \Gamma\left(k+\frac{1}{2}\right)}\right. \\
& \left.\times \frac{(\delta / 2)^{k} \exp (-\delta / 2)}{\Gamma(k+1)}\right\}, \quad \text { for } y>0
\end{aligned}
$$

The $r$ th moment, the expected value, the variance, and the mean squared error of $\hat{C}_{\mathrm{ia}}$, therefore, can be calculated as

$$
\begin{aligned}
\mathrm{E}\left(\hat{C}_{\mathrm{ia}}^{r}\right)= & \left(\frac{C_{\mathrm{ip}}}{n}\right)^{r} \mathrm{E}\left[\chi_{1}^{2}(\delta)\right]^{r} \\
= & \sum_{k=0}^{\infty}\left\{\left(\frac{2 C_{\mathrm{ip}}}{n}\right)^{r} \frac{\Gamma\left(k+\frac{1}{2}+r\right)}{\Gamma\left(k+\frac{1}{2}\right)}\right. \\
& \left.\times \frac{(\delta / 2)^{k} \exp (-\delta / 2)}{\Gamma(k+1)}\right\} \\
\mathrm{E}\left(\hat{C}_{\mathrm{ia}}\right)= & \left(\frac{C_{\mathrm{ip}}}{n}\right) \mathrm{E}\left[\chi_{1}^{2}(\delta)\right] \\
= & \left(\frac{C_{\mathrm{ip}}}{n}\right)(1+\delta) \\
= & \frac{C_{\mathrm{ip}}}{n}+C_{\mathrm{ia}}
\end{aligned}
$$

Copyright $\odot 2001$ John Wiley \& Sons, Ltd.

$$
\begin{aligned}
\operatorname{Var}\left(\hat{C}_{\mathrm{ia}}\right) & =\left(\frac{C_{\mathrm{ip}}}{n}\right)^{2} \operatorname{Var}\left[\chi_{1}^{2}(\delta)\right] \\
& =\left(\frac{C_{\mathrm{ip}}}{n}\right)^{2}(2+4 \delta) \\
& =\frac{4 C_{\mathrm{ip}} C_{\mathrm{ia}}}{n}+\frac{2 C_{\mathrm{ip}}^{2}}{n} \\
\operatorname{MSE}\left(\hat{C}_{\mathrm{ia}}\right) & =\operatorname{Var}\left(\hat{C}_{\mathrm{ia}}\right)+\left[\mathrm{E}\left(\hat{C}_{\mathrm{ia}}\right)-C_{\mathrm{ia}}\right]^{2} \\
& =\frac{4 C_{\mathrm{ip}} C_{\mathrm{ia}}}{n}+\frac{3 C_{\mathrm{ip}}^{2}}{n^{2}}
\end{aligned}
$$

Since $\bar{X}$ is the MLE of $\mu$, then by the invariance property of the MLE, the natural estimator $\hat{C}_{\mathrm{ia}}$ is the MLE of $C_{\mathrm{ia}}$. Noting that $\mathrm{E}\left(\hat{C}_{\mathrm{ia}}\right)=C_{\mathrm{ia}}+$ $\left(C_{\mathrm{ip}} / n\right)$, and $\mathrm{E}\left(\hat{C}_{\mathrm{ip}}\right)=C_{\mathrm{ip}}$, the corrected estimator $\tilde{C}_{\mathrm{ia}}=\hat{C}_{\mathrm{ia}}-\left(\hat{C}_{\mathrm{ip}} / n\right)$ must be unbiased for $C_{\mathrm{ia}}$. We can show that $\tilde{C}_{\mathrm{ia}}$ is the UMVUE of $C_{\mathrm{ia}}$, which is consistent. We can also show that $\sqrt{n}\left(\tilde{C}_{\mathrm{ia}}-C_{\mathrm{ia}}\right)$ converges to $N\left(0,4 C_{\mathrm{ip}} C_{\mathrm{ia}}\right)$ in distribution, and $\tilde{C}_{\mathrm{ia}}$ is asymptotically efficient (see the Appendix for the proofs). Thus, in real-world applications using the UMVUE $\tilde{C}_{\text {ia }}$, which has all the desired statistical properties, as an estimate of $C_{\mathrm{ia}}$ would be reasonable.

We note that the MLE $\hat{C}_{\text {ia }}$ has smaller variance than the UMVUE $\tilde{C}_{\text {ia }}$. However, we can show that $\operatorname{MSE}\left(\tilde{C}_{\mathrm{ia}}\right)=4 C_{\mathrm{ip}} C_{\mathrm{ia}} / n+[2 / n(n-1)]\left(C_{\mathrm{ip}}\right)^{2}$, and so $\operatorname{MSE}\left(\tilde{C}_{\mathrm{ia}}\right)-\operatorname{MSE}\left(\hat{C}_{\mathrm{ia}}\right)=\left[(3-n) / n^{2}(n-1)\right]\left(C_{\mathrm{ip}}\right)^{2}$, which is greater than 0 for $n=2$, equal to 0 for $n=3$, and less than 0 for $n \geq 4$. Therefore, the UMVUE $\tilde{C}_{\mathrm{ia}}$ has smaller mean squared error than the MLE $\hat{C}_{\mathrm{ia}}$, and is more reliable for applications with $n \geq 4$.

Tables 3(a) and 3(b) display the relative error, $\left[\operatorname{MSE}_{\mathrm{R}}\left(\tilde{C}_{\mathrm{ia}}\right)\right]^{1 / 2}$, of the UMVUE $\tilde{C}_{\mathrm{ia}}$ for $C_{\mathrm{ip}}=1.00$, $0.56,0.44,0.36,0.25$, and $C_{\mathrm{ia}}=2.25$. The value of $C_{\mathrm{ia}}$ is equivalent to $C_{\mathrm{a}}=0.50$. The relative errors, $\left[\operatorname{MSE}_{\mathrm{R}}\left(\tilde{C}_{\mathrm{ia}}\right)\right]^{1 / 2}$, for $C_{\mathrm{ia}}=5.06$ and 0.56 are available from the authors. We note that if the process is perfectly centered, then $C_{\mathrm{ia}}=0.00$ (equivalently, $\left.C_{\mathrm{a}}=1.00\right)$. For example, for $C_{\mathrm{ip}}=1.00, C_{\mathrm{ia}}=2.25$, and $n=300$ we have $\left[\operatorname{MSE}_{\mathrm{R}}\left(\tilde{C}_{\mathrm{ia}}\right)\right]^{1 / 2}=0.0770$. Thus, the average error (average relative deviation) of $\tilde{C}_{\mathrm{ia}}$ would be no greater than $7.70 \%$ of the true $C_{\text {ia }}$. Tables 4(a) and 4(b) display the relative error, $\left[\operatorname{MSE}{ }_{\mathrm{R}}\left(\hat{C}_{\mathrm{ia}}\right)\right]^{1 / 2}$, of the MLE $\hat{C}_{\mathrm{ia}}$ for $C_{\mathrm{ip}}=1.00$, $0.56,0.44,0.36,0.25$, and $C_{\mathrm{ia}}=2.25$ (tables of $\left[\operatorname{MSE}_{\mathrm{R}}\left(\hat{C}_{\mathrm{ia}}\right)\right]^{1 / 2}$ for other values of $C_{\mathrm{ia}}$ are available from the authors). We note that for $n<30$, the difference between the two relative errors (percentage of deviations) is significant. However, for $n>30$, the difference between the two is negligible (less than $0.3 \%$ ), and using either of the two estimators is equally reliable.

Qual. Reliab. Engng. Int. 2001; 17: 279-290 
Table 3. $\left[\operatorname{MSE}_{\mathrm{R}}\left(\tilde{C}_{\mathrm{ia}}\right)\right]^{1 / 2}$ for various $C_{\mathrm{ip}}, C_{\mathrm{ia}}=2.25$ and (a) $n=2(1) 50$ and (b) $n=60(10) 550$

\begin{tabular}{|c|c|c|c|c|c|c|c|c|c|c|c|}
\hline \multirow[b]{2}{*}{$n$} & \multicolumn{5}{|c|}{$C_{\text {ip }}$} & \multirow[b]{2}{*}{$n$} & \multicolumn{5}{|c|}{$C_{\mathrm{ip}}$} \\
\hline & 1.00 & 0.56 & 0.44 & 0.36 & 0.25 & & 1.00 & 0.56 & 0.44 & 0.36 & 0.25 \\
\hline 1 & $* * * * * *$ & $* * * * * *$ & $* * * * * *$ & $* * * * * *$ & $* * * * * *$ & 60 & 0.1725 & 0.1292 & 0.1149 & 0.1034 & 0.0861 \\
\hline 2 & 1.0423 & 0.7500 & 0.6588 & 0.5879 & 0.4843 & 70 & 0.1596 & 0.1196 & 0.1063 & 0.0957 & 0.0797 \\
\hline 3 & 0.8114 & 0.5951 & 0.5257 & 0.4710 & 0.3902 & 80 & 0.1493 & 0.1119 & 0.0994 & 0.0895 & 0.0746 \\
\hline 4 & 0.6909 & 0.5103 & 0.4517 & 0.4053 & 0.3364 & 90 & 0.1407 & 0.1055 & 0.0937 & 0.0844 & 0.0703 \\
\hline 5 & 0.6126 & 0.4541 & 0.4024 & 0.3613 & 0.3002 & 100 & 0.1335 & 0.1001 & 0.0889 & 0.0800 & 0.0667 \\
\hline 6 & 0.5563 & 0.4133 & 0.3665 & 0.3292 & 0.2737 & 110 & 0.1273 & 0.0954 & 0.0848 & 0.0763 & 0.0636 \\
\hline 7 & 0.5132 & 0.3819 & 0.3387 & 0.3044 & 0.2531 & 120 & 0.1218 & 0.0913 & 0.0812 & 0.0731 & 0.0609 \\
\hline 8 & 0.4788 & 0.3567 & 0.3165 & 0.2845 & 0.2366 & 130 & 0.1170 & 0.0877 & 0.0780 & 0.0702 & 0.0585 \\
\hline 9 & 0.4506 & 0.3359 & 0.2981 & 0.2680 & 0.2230 & 140 & 0.1128 & 0.0846 & 0.0752 & 0.0676 & 0.0564 \\
\hline 10 & 0.4268 & 0.3184 & 0.2826 & 0.2541 & 0.2115 & 150 & 0.1089 & 0.0817 & 0.0726 & 0.0653 & 0.0544 \\
\hline 11 & 0.4065 & 0.3034 & 0.2693 & 0.2422 & 0.2016 & 160 & 0.1055 & 0.0791 & 0.0703 & 0.0633 & 0.0527 \\
\hline 12 & 0.3888 & 0.2903 & 0.2578 & 0.2318 & 0.1929 & 170 & 0.1023 & 0.0767 & 0.0682 & 0.0614 & 0.0511 \\
\hline 13 & 0.3732 & 0.2788 & 0.2475 & 0.2226 & 0.1853 & 180 & 0.0994 & 0.0746 & 0.0663 & 0.0596 & 0.0497 \\
\hline 14 & 0.3594 & 0.2685 & 0.2385 & 0.2145 & 0.1786 & 190 & 0.0968 & 0.0726 & 0.0645 & 0.0581 & 0.0484 \\
\hline 15 & 0.3470 & 0.2593 & 0.2303 & 0.2071 & 0.1725 & 200 & 0.0943 & 0.0707 & 0.0629 & 0.0566 & 0.0471 \\
\hline 16 & 0.3358 & 0.2510 & 0.2230 & 0.2005 & 0.1670 & 210 & 0.0921 & 0.0690 & 0.0614 & 0.0552 & 0.0460 \\
\hline 17 & 0.3256 & 0.2435 & 0.2163 & 0.1945 & 0.1620 & 220 & 0.0899 & 0.0674 & 0.0599 & 0.0539 & 0.0450 \\
\hline 18 & 0.3163 & 0.2366 & 0.2101 & 0.1890 & 0.1574 & 230 & 0.0880 & 0.0660 & 0.0586 & 0.0528 & 0.0440 \\
\hline 19 & 0.3078 & 0.2302 & 0.2045 & 0.1839 & 0.1532 & 240 & 0.0861 & 0.0646 & 0.0574 & 0.0516 & 0.0430 \\
\hline 20 & 0.2999 & 0.2243 & 0.1993 & 0.1793 & 0.1493 & 250 & 0.0844 & 0.0633 & 0.0562 & 0.0506 & 0.0422 \\
\hline 21 & 0.2926 & 0.2189 & 0.1945 & 0.1749 & 0.1457 & 260 & 0.0827 & 0.0620 & 0.0551 & 0.0496 & 0.0413 \\
\hline 22 & 0.2858 & 0.2138 & 0.1900 & 0.1709 & 0.1423 & 270 & 0.0812 & 0.0609 & 0.0541 & 0.0487 & 0.0406 \\
\hline 23 & 0.2794 & 0.2091 & 0.1858 & 0.1671 & 0.1392 & 280 & 0.0797 & 0.0598 & 0.0531 & 0.0478 & 0.0398 \\
\hline 24 & 0.2735 & 0.2047 & 0.1818 & 0.1636 & 0.1362 & 290 & 0.0783 & 0.0587 & 0.0522 & 0.0470 & 0.0392 \\
\hline 25 & 0.2679 & 0.2005 & 0.1781 & 0.1603 & 0.1335 & 300 & 0.0770 & 0.0577 & 0.0513 & 0.0462 & 0.0385 \\
\hline 26 & 0.2626 & 0.1966 & 0.1747 & 0.1571 & 0.1309 & 310 & 0.0758 & 0.0568 & 0.0505 & 0.0454 & 0.0379 \\
\hline 27 & 0.2577 & 0.1929 & 0.1714 & 0.1542 & 0.1284 & 320 & 0.0746 & 0.0559 & 0.0497 & 0.0447 & 0.0373 \\
\hline 28 & 0.2530 & 0.1894 & 0.1683 & 0.1514 & 0.1261 & 330 & 0.0734 & 0.0551 & 0.0489 & 0.0440 & 0.0367 \\
\hline 29 & 0.2486 & 0.1861 & 0.1654 & 0.1488 & 0.1239 & 340 & 0.0723 & 0.0542 & 0.0482 & 0.0434 & 0.0362 \\
\hline 30 & 0.2444 & 0.1830 & 0.1626 & 0.1463 & 0.1218 & 350 & 0.0713 & 0.0535 & 0.0475 & 0.0428 & 0.0356 \\
\hline 31 & 0.2404 & 0.1800 & 0.1599 & 0.1439 & 0.1198 & 360 & 0.0703 & 0.0527 & 0.0469 & 0.0422 & 0.0351 \\
\hline 32 & 0.2365 & 0.1771 & 0.1574 & 0.1416 & 0.1180 & 370 & 0.0693 & 0.0520 & 0.0462 & 0.0416 & 0.0347 \\
\hline 33 & 0.2329 & 0.1744 & 0.1550 & 0.1394 & 0.1162 & 380 & 0.0684 & 0.0513 & 0.0456 & 0.0410 & 0.0342 \\
\hline 34 & 0.2294 & 0.1718 & 0.1527 & 0.1374 & 0.1144 & 390 & 0.0675 & 0.0506 & 0.0450 & 0.0405 & 0.0338 \\
\hline 35 & 0.2261 & 0.1693 & 0.1505 & 0.1354 & 0.1128 & 400 & 0.0667 & 0.0500 & 0.0444 & 0.0400 & 0.0333 \\
\hline 36 & 0.2229 & 0.1670 & 0.1484 & 0.1335 & 0.1112 & 410 & 0.0659 & 0.0494 & 0.0439 & 0.0395 & 0.0329 \\
\hline 37 & 0.2199 & 0.1647 & 0.1463 & 0.1317 & 0.1097 & 420 & 0.0651 & 0.0488 & 0.0434 & 0.0390 & 0.0325 \\
\hline 38 & 0.2169 & 0.1625 & 0.1444 & 0.1299 & 0.1082 & 430 & 0.0643 & 0.0482 & 0.0429 & 0.0386 & 0.0322 \\
\hline 39 & 0.2141 & 0.1604 & 0.1425 & 0.1282 & 0.1068 & 440 & 0.0636 & 0.0477 & 0.0424 & 0.0381 & 0.0318 \\
\hline 40 & 0.2114 & 0.1584 & 0.1407 & 0.1266 & 0.1055 & 450 & 0.0629 & 0.0471 & 0.0419 & 0.0377 & 0.0314 \\
\hline 41 & 0.2088 & 0.1564 & 0.1390 & 0.1251 & 0.1042 & 460 & 0.0622 & 0.0466 & 0.0414 & 0.0373 & 0.0311 \\
\hline 42 & 0.2063 & 0.1545 & 0.1373 & 0.1236 & 0.1029 & 470 & 0.0615 & 0.0461 & 0.0410 & 0.0369 & 0.0308 \\
\hline 43 & 0.2039 & 0.1527 & 0.1357 & 0.1221 & 0.1017 & 480 & 0.0609 & 0.0456 & 0.0406 & 0.0365 & 0.0304 \\
\hline 44 & 0.2015 & 0.1510 & 0.1342 & 0.1207 & 0.1006 & 490 & 0.0602 & 0.0452 & 0.0402 & 0.0361 & 0.0301 \\
\hline 45 & 0.1993 & 0.1493 & 0.1327 & 0.1194 & 0.0994 & 500 & 0.0596 & 0.0447 & 0.0398 & 0.0358 & 0.0298 \\
\hline 46 & 0.1971 & 0.1476 & 0.1312 & 0.1181 & 0.0984 & 510 & 0.0591 & 0.0443 & 0.0394 & 0.0354 & 0.0295 \\
\hline 47 & 0.1950 & 0.1461 & 0.1298 & 0.1168 & 0.0973 & 520 & 0.0585 & 0.0439 & 0.0390 & 0.0351 & 0.0292 \\
\hline 48 & 0.1929 & 0.1445 & 0.1284 & 0.1156 & 0.0963 & 530 & 0.0579 & 0.0434 & 0.0386 & 0.0348 & 0.0290 \\
\hline 49 & 0.1909 & 0.1430 & 0.1271 & 0.1144 & 0.0953 & 540 & 0.0574 & 0.0430 & 0.0383 & 0.0344 & 0.0287 \\
\hline 50 & 0.1890 & 0.1416 & 0.1258 & 0.1132 & 0.0943 & 550 & 0.0569 & 0.0426 & 0.0379 & 0.0341 & 0.0284 \\
\hline
\end{tabular}


Table 4. $\left[\operatorname{MSE} \mathrm{R}\left(\hat{C}_{\mathrm{ia}}\right)\right]^{1 / 2}$ for various $C_{\mathrm{ip}}, C_{\mathrm{ia}}=2.25$ and (a) $n=2(1) 50$ and (b) $n=60(10) 550$

\begin{tabular}{|c|c|c|c|c|c|c|c|c|c|c|c|}
\hline \multirow[b]{2}{*}{$n$} & \multicolumn{5}{|c|}{$C_{\mathrm{ip}}$} & \multirow[b]{2}{*}{$n$} & \multicolumn{5}{|c|}{$C_{\mathrm{ip}}$} \\
\hline & 1.00 & 0.56 & 0.44 & 0.36 & 0.25 & & 1.00 & 0.56 & 0.44 & 0.36 & 0.25 \\
\hline 1 & 1.5396 & 1.0897 & 0.9525 & 0.8466 & 0.6939 & 60 & 0.1726 & 0.1293 & 0.1149 & 0.1034 & 0.0861 \\
\hline 2 & 1.0184 & 0.7395 & 0.6514 & 0.5824 & 0.4811 & 70 & 0.1597 & 0.1197 & 0.1064 & 0.0957 & 0.0797 \\
\hline 3 & 0.8114 & 0.5951 & 0.5257 & 0.4710 & 0.3902 & 80 & 0.1494 & 0.1119 & 0.0995 & 0.0895 & 0.0746 \\
\hline 4 & 0.6939 & 0.5116 & 0.4526 & 0.4060 & 0.3368 & 90 & 0.1408 & 0.1055 & 0.0938 & 0.0844 & 0.0703 \\
\hline 5 & 0.6158 & 0.4555 & 0.4034 & 0.3620 & 0.3006 & 100 & 0.1336 & 0.1001 & 0.0890 & 0.0800 & 0.0667 \\
\hline 6 & 0.5592 & 0.4146 & 0.3673 & 0.3298 & 0.2740 & 110 & 0.1273 & 0.0954 & 0.0848 & 0.0763 & 0.0636 \\
\hline 7 & 0.5158 & 0.3830 & 0.3395 & 0.3050 & 0.2535 & 120 & 0.1219 & 0.0914 & 0.0812 & 0.0731 & 0.0609 \\
\hline 8 & 0.4811 & 0.3577 & 0.3172 & 0.2850 & 0.2369 & 130 & 0.1171 & 0.0878 & 0.0780 & 0.0702 & 0.0585 \\
\hline 9 & 0.4526 & 0.3368 & 0.2987 & 0.2684 & 0.2232 & 140 & 0.1128 & 0.0846 & 0.0752 & 0.0676 & 0.0564 \\
\hline 10 & 0.4286 & 0.3192 & 0.2832 & 0.2545 & 0.2117 & 150 & 0.1090 & 0.0817 & 0.0726 & 0.0653 & 0.0544 \\
\hline 11 & 0.4081 & 0.3041 & 0.2698 & 0.2525 & 0.2018 & 160 & 0.1055 & 0.0791 & 0.0703 & 0.0633 & 0.0527 \\
\hline 12 & 0.3902 & 0.2909 & 0.2582 & 0.2321 & 0.1931 & 170 & 0.1024 & 0.0767 & 0.0682 & 0.0614 & 0.0511 \\
\hline 13 & 0.3745 & 0.2793 & 0.2479 & 0.2229 & 0.1855 & 180 & 0.0995 & 0.0746 & 0.0663 & 0.0596 & 0.0497 \\
\hline 14 & 0.3606 & 0.2690 & 0.2388 & 0.2147 & 0.1787 & 190 & 0.0968 & 0.0726 & 0.0645 & 0.0581 & 0.0484 \\
\hline 15 & 0.3481 & 0.2598 & 0.2306 & 0.2074 & 0.1726 & 200 & 0.0944 & 0.0707 & 0.0629 & 0.0566 & 0.0472 \\
\hline 16 & 0.3368 & 0.2515 & 0.2232 & 0.2007 & 0.1671 & 210 & 0.0921 & 0.0690 & 0.0614 & 0.0552 & 0.0460 \\
\hline 17 & 0.3265 & 0.2439 & 0.2165 & 0.1947 & 0.1621 & 220 & 0.0900 & 0.0674 & 0.0599 & 0.0540 & 0.0450 \\
\hline 18 & 0.3172 & 0.2369 & 0.2104 & 0.1892 & 0.1575 & 230 & 0.0880 & 0.0660 & 0.0586 & 0.0528 & 0.0440 \\
\hline 19 & 0.3086 & 0.2305 & 0.2047 & 0.1841 & 0.1533 & 240 & 0.0861 & 0.0646 & 0.0574 & 0.0517 & 0.0430 \\
\hline 20 & 0.3006 & 0.2247 & 0.1995 & 0.1794 & 0.1494 & 250 & 0.0844 & 0.0633 & 0.0562 & 0.0506 & 0.0422 \\
\hline 21 & 0.2933 & 0.2192 & 0.1947 & 0.1751 & 0.1458 & 260 & 0.0827 & 0.0620 & 0.0551 & 0.0496 & 0.0414 \\
\hline 22 & 0.2864 & 0.2141 & 0.1901 & 0.1710 & 0.1424 & 270 & 0.0812 & 0.0609 & 0.0541 & 0.0487 & 0.0406 \\
\hline 23 & 0.2800 & 0.2094 & 0.1859 & 0.1672 & 0.1393 & 280 & 0.0797 & 0.0598 & 0.0531 & 0.0478 & 0.0398 \\
\hline 24 & 0.2740 & 0.2049 & 0.1820 & 0.1637 & 0.1363 & 290 & 0.0783 & 0.0587 & 0.0522 & 0.0470 & 0.0392 \\
\hline 25 & 0.2684 & 0.2007 & 0.1783 & 0.1604 & 0.13 & 300 & 0.0770 & 0.0578 & 0.0513 & 0.0462 & 0.0385 \\
\hline 26 & 0.2632 & 0.1968 & 0.1748 & 0.1573 & & 31 & 0.0758 & 0.0568 & 0.0505 & 0.0454 & 0.0379 \\
\hline 27 & 0.2582 & 0.1931 & 0.1715 & 0.1543 & 0.1285 & 320 & 0.0746 & 0.0559 & 0.0497 & 0.0447 & 0.0373 \\
\hline 28 & 0.2535 & 0.1896 & 0.1684 & 0.1515 & 0.1262 & 330 & 0.0734 & 0.0551 & 0.0489 & 0.0440 & 0.0367 \\
\hline 29 & 0.2490 & 0.1863 & 0.1655 & 0.1489 & 0.1240 & 340 & 0.0723 & 0.0542 & 0.0482 & 0.0434 & 0.0362 \\
\hline 30 & 0.2448 & 0.1831 & 0.1627 & 0.1464 & 0.1219 & 350 & 0.0713 & 0.0535 & 0.0475 & 0.0428 & 0.0356 \\
\hline 31 & 0.2408 & 0.1801 & 0.1600 & 0.1440 & 0.1199 & 360 & 0.0703 & 0.0527 & 0.0469 & 0.0422 & 0.0351 \\
\hline 32 & 0.2369 & 0.1773 & 0.1575 & 0.1417 & 0.1180 & 370 & 0.0693 & 0.0520 & 0.0462 & 0.0416 & 0.0347 \\
\hline 33 & 0.2333 & 0.1746 & 0.1551 & 0.1395 & 0.1162 & 380 & 0.0684 & 0.0513 & 0.0456 & 0.0410 & 0.0342 \\
\hline 34 & 0.2298 & 0.1720 & 0.1528 & 0.1374 & 0.1145 & 390 & 0.0675 & 0.0506 & 0.0450 & 0.0405 & 0.0338 \\
\hline 35 & 0.2264 & 0.1695 & 0.1506 & 0.1355 & 0.1128 & 400 & 0.0667 & 0.0500 & 0.0445 & 0.0400 & 0.0333 \\
\hline 36 & 0.2232 & 0.1671 & 0.1485 & 0.1336 & 0.1112 & 410 & 0.0659 & 0.0494 & 0.0439 & 0.0395 & 0.0329 \\
\hline 37 & 0.2202 & 0.1648 & 0.1464 & 0.1317 & 0.1097 & 420 & 0.0651 & 0.0488 & 0.0434 & 0.0390 & 0.0325 \\
\hline 38 & 0.2172 & 0.1626 & 0.1445 & 0.1300 & 0.1083 & 430 & 0.0643 & 0.0482 & 0.0429 & 0.0386 & 0.0322 \\
\hline 39 & 0.2144 & 0.1605 & 0.1426 & 0.1283 & 0.1069 & 440 & 0.0636 & 0.0477 & 0.0424 & 0.0381 & 0.0318 \\
\hline 40 & 0.2117 & 0.1585 & 0.1408 & 0.1267 & 0.1055 & 450 & 0.0629 & 0.0415 & 0.0419 & 0.0377 & 0.0314 \\
\hline 41 & 0.2091 & 0.1565 & 0.1391 & 0.1251 & 0.10 & 460 & 0.0622 & 0.0466 & 0.0415 & 0.0373 & 0.0311 \\
\hline 42 & 0.2066 & 0.1546 & 0.1374 & 0.1236 & 0.1030 & 47 & 0.0615 & 0.0461 & 0.0410 & 0.0369 & 0.0308 \\
\hline 43 & 0.2041 & 0.1528 & 0.1358 & 0.1222 & 0.1018 & 48 & 0.0609 & 0.0457 & 0.0406 & 0.0365 & 0.0304 \\
\hline 44 & 0.2018 & 0.1511 & 0.1342 & 0.1208 & 0.1006 & 490 & 0.0603 & 0.0452 & 0.0402 & 0.0361 & 0.0301 \\
\hline 45 & 0.1995 & 0.1494 & 0.1327 & 0.1194 & 0.0995 & 500 & 0.0596 & 0.0447 & 0.0398 & 0.0358 & 0.0298 \\
\hline 46 & 0.1973 & 0.1477 & 0.1313 & 0.1181 & 0.0984 & 510 & 0.0591 & 0.0443 & 0.0394 & 0.0354 & 0.0295 \\
\hline 47 & 0.1952 & 0.1462 & 0.1299 & 0.1168 & 0.0973 & 520 & 0.0585 & 0.0439 & 0.0390 & 0.0351 & 0.0292 \\
\hline 48 & 0.1931 & 0.1446 & 0.1285 & 0.1156 & 0.0963 & 530 & 0.0579 & 0.0434 & 0.0386 & 0.0348 & 0.0290 \\
\hline 49 & 0.1911 & 0.1431 & 0.1272 & 0.1144 & 0.0953 & 540 & 0.0574 & 0.0430 & 0.0383 & 0.0344 & 0.0287 \\
\hline 50 & 0.1892 & 0.1417 & 0.1259 & 0.1133 & 0.0944 & 550 & 0.0569 & 0.0426 & 0.0379 & 0.0341 & 0.0284 \\
\hline
\end{tabular}




\section{ESTIMATING PROCESS INCAPABILITY}

To estimate the process incapability (a combined measure of process imprecision and process inaccuracy), we consider the natural estimator $\hat{C}_{\mathrm{pp}}$ defined as the following, where $\bar{X}=\sum_{i=1}^{n} X_{i} / n$, which can also be written as a function of $C_{\mathrm{ip}}$ :

$$
\begin{aligned}
\hat{C}_{\mathrm{pp}} & =\frac{1}{n} \sum_{i=1}^{n} \frac{\left(X_{i}-\bar{X}\right)^{2}}{D^{2}}+\frac{(\bar{X}-T)^{2}}{D^{2}} \\
& =\frac{C_{\mathrm{ip}}}{n} \frac{n \hat{C}_{\mathrm{pp}}}{C_{\mathrm{ip}}} \\
& =\frac{C_{\mathrm{ip}}}{n} \sum_{i=1}^{n} \frac{\left(X_{i}-T\right)^{2}}{\sigma^{2}}
\end{aligned}
$$

If the process characteristic is normally distributed, then the estimator $\hat{C}_{\mathrm{pp}}$ is distributed as $\left[C_{\mathrm{ip}} / n\right] \chi_{n}^{2}(\delta)$, where $\chi_{n}^{2}(\delta)$ is a non-central chi-squared distribution with $n$ degrees of freedom and non-centrality parameter $\delta=n(\mu-T)^{2} / \sigma^{2}=n C_{\mathrm{ia}} / C_{\mathrm{ip}}$. Therefore, the probability density function of $\hat{C}_{\mathrm{pp}}$ can be expressed as

$$
\begin{aligned}
h(y)= & \sum_{k=0}^{\infty}\left\{\frac{\left[(n y) /\left(2 C_{\mathrm{ip}}\right)\right]^{k+(n / 2)} \exp \left[-(n y) /\left(2 C_{\mathrm{ip}}\right)\right]}{y \Gamma(k+(n / 2))}\right. \\
& \left.\times \frac{(\delta / 2)^{k} \exp (-\delta / 2)}{\Gamma(k+1)}\right\}, \quad \text { for } y>0
\end{aligned}
$$

The $r$ th moment (hence the expected value, the variance, and the mean squared error) of $\hat{C}_{\mathrm{pp}}$, therefore can be calculated as follows:

$$
\begin{aligned}
& \mathrm{E}\left(\hat{C}_{\mathrm{pp}}^{r}\right)=\left(\frac{C_{\mathrm{ip}}}{n}\right)^{r} \mathrm{E}\left[\chi_{n}^{2}(\delta)\right]^{r} \\
&= \sum_{k=0}^{\infty}\left\{\left(\frac{2 C_{\mathrm{ip}}}{n}\right)^{r} \frac{\Gamma(k+(n / 2)+r)}{\Gamma(k+(n / 2))}\right. \\
&\left.\times \frac{(\delta / 2)^{k} \exp (-\delta / 2)}{\Gamma(k+1)}\right\} \\
& \mathrm{E}\left(\hat{C}_{\mathrm{pp}}\right)=\left(\frac{C_{\mathrm{ip}}}{n}\right) \mathrm{E}\left[\chi_{n}^{2}(\delta)\right] \\
&= \frac{C_{\mathrm{ip}}}{n}(n+\delta) \\
&= C_{\mathrm{ip}}+C_{\mathrm{ia}}=C_{\mathrm{pp}} \\
& \operatorname{Var}\left(\hat{C}_{\mathrm{pp}}\right)=\left(\frac{C_{\mathrm{ip}}}{n}\right)^{2} \operatorname{Var}\left[\chi_{n}^{2}(\delta)\right] \\
&=\left(\frac{C_{\mathrm{ip}}}{n}\right)^{2}(2 n+4 \delta) \\
&=\frac{2 C_{\mathrm{ip}}}{n}\left(C_{\mathrm{ia}}+C_{\mathrm{pp}}\right)
\end{aligned}
$$

Copyright $\odot 2001$ John Wiley \& Sons, Ltd.

$$
\begin{aligned}
\operatorname{MSE}\left(\hat{C}_{\mathrm{pp}}\right) & =\operatorname{Var}\left(\hat{C}_{\mathrm{pp}}\right)+\left[\mathrm{E}\left(\hat{C}_{\mathrm{pp}}\right)-C_{\mathrm{pp}}\right]^{2} \\
& =\frac{2 C_{\mathrm{ip}}}{n}\left(C_{\mathrm{ia}}+C_{\mathrm{pp}}\right)
\end{aligned}
$$

If the process characteristic follows the normal distribution, then we can show that $\hat{C}_{\mathrm{pp}}$ is the MLE, which is also the UMVUE of $C_{\mathrm{pp}}$. We can also show that $\hat{C}_{\mathrm{pp}}$ is consistent, $\sqrt{n}\left(\hat{C}_{\mathrm{pp}}-C_{\mathrm{pp}}\right)$ converges to $N\left(0,2 C_{\mathrm{ip}} C_{\mathrm{ia}}+2 C_{\mathrm{ip}} C_{\mathrm{pp}}\right)$ in distribution, and $\hat{C}_{\mathrm{pp}}$ is asymptotically efficient (see the Appendix for the proofs). Since the estimator has all the desired statistical properties, in practice using $\hat{C}_{\mathrm{pp}}$ to estimate process incapability would be reasonable.

\section{DECISION MAKING}

Under the normality assumption, $n \hat{C}_{\mathrm{pp}} /\left(C_{\mathrm{pp}}-C_{\mathrm{ia}}\right)$ is distributed as $\chi_{n}^{2}(\delta)$, a non-central chi-squared distribution with $n$ degrees of freedom and noncentrality parameter $\delta=n(\mu-T)^{2} / \sigma^{2}=n C_{\text {ia }} / C_{\text {ip }}$. Let $W=W\left(X_{1}, X_{2}, \ldots, X_{n}\right)$ be a statistic calculated from the sample data satisfying $\mathrm{P}\left\{C_{\mathrm{pp}} \leq W\right\}=1-\alpha$, where the confidence level $1-\alpha$ does not depend on $C_{\mathrm{pp}}$. Then, $W$ is a $100(1-\alpha) \%$ upper confidence limit for $C_{\mathrm{pp}}$. We note that

$$
\begin{aligned}
\mathrm{P}\left\{C_{\mathrm{pp}}\right. & \leq W\} \\
& =\mathrm{P}\left\{C_{\mathrm{pp}}-C_{\mathrm{ia}} \leq W-C_{\mathrm{ia}}\right\} \\
& =\mathrm{P}\left\{1 /\left(C_{\mathrm{pp}}-C_{\mathrm{ia}}\right) \geq 1 /\left(W-C_{\mathrm{ia}}\right)\right\} \\
& =\mathrm{P}\left\{n \hat{C}_{\mathrm{pp}} /\left(\hat{C}_{\mathrm{pp}}-C_{\mathrm{ia}}\right) \geq n \hat{C}_{\mathrm{pp}} /\left(W-C_{\mathrm{ia}}\right)\right\} \\
& =\mathrm{P}\left\{\chi_{n}^{2}(\delta) \geq n \hat{C}_{\mathrm{pp}} /\left(W-C_{\mathrm{ia}}\right)\right\} \\
& =1-\alpha
\end{aligned}
$$

Therefore, $n \hat{C}_{\mathrm{pp}} /\left(W-C_{\mathrm{ia}}\right)=\chi_{n}^{2}(\alpha, \delta)$, where $\chi_{n}^{2}(\alpha, \delta)$ is the (lower) $\alpha$ th percentile of the $\chi_{n}^{2}(\delta)$ distribution. A $100(1-\alpha) \%$ upper confidence limit on $C_{\mathrm{pp}}$ can be written in terms of $\hat{C}_{\mathrm{pp}}$ as $W=C_{\mathrm{ia}}+$ $\left[n \hat{C}_{\mathrm{pp}} / \chi_{n}^{2}(\alpha, \delta)\right]$.

Tables 5(a), 6(a), and 7(a) give 90\%, 95\%, and 99\% upper confidence limits for $C_{\mathrm{pp}}$ under $\mu=T$ with $n$ given, and $\hat{C}_{\mathrm{pp}}$ calculated from the sample data. On the other hand, $\hat{C}_{\mathrm{pp}}=\chi_{n}^{2}(\alpha, \delta)\left(W-C_{\mathrm{ia}}\right) / n$ depends on $W$ and $C_{\text {ia }}$. In the special case when $\mu=T$ and $W$ equals the recommended maximum value for $C_{\mathrm{pp}}$, the probability that $C_{\mathrm{pp}} \leq W$ would be either 1 or 0 if $C_{\mathrm{pp}}$ were known. In practice, since $C_{\mathrm{pp}}$ is unknown, we take a random sample of size $n$ and calculate $\hat{C}_{\mathrm{pp}}$.

Suppose that a process is capable if $\hat{C}_{\mathrm{pp}} \leq$ $\chi_{n}^{2}(\alpha, \delta)\left(C_{0}-C_{\mathrm{ia}}\right) / n$, where $C_{0}$ is the recommended maximum value, and we claim that the process is capable for at least $100(1-\alpha) \%$ of the time. Therefore, 
Table 5. (a) The $90 \%$ upper confidence limits for $C_{\mathrm{pp}}$ under $\mu=T$, with given $\hat{C}_{\mathrm{pp}}$. (b) The maximum value of $\hat{C}_{\mathrm{pp}}$ under $\mu=T$ for which the process is capable $\left(C_{\mathrm{pp}} \leq C_{0}\right) 90 \%$ of the time

(a)

\begin{tabular}{ccccccccccccc}
\hline & \multicolumn{10}{c}{ Sample size $n$} \\
\cline { 2 - 14 }$\hat{C}_{\mathrm{pp}}$ & 5 & 10 & 15 & 20 & 25 & 30 & 35 & 40 & 45 & 50 & 55 & 60 \\
\hline 0.25 & 0.776 & 0.514 & 0.439 & 0.402 & 0.379 & 0.364 & 0.353 & 0.344 & 0.337 & 0.332 & 0.327 & 0.323 \\
0.36 & 1.118 & 0.740 & 0.632 & 0.579 & 0.546 & 0.524 & 0.508 & 0.496 & 0.486 & 0.478 & 0.471 & 0.465 \\
0.44 & 1.366 & 0.904 & 0.772 & 0.707 & 0.668 & 0.641 & 0.621 & 0.606 & 0.594 & 0.584 & 0.575 & 0.568 \\
0.56 & 1.739 & 1.151 & 0.983 & 0.900 & 0.850 & 0.816 & 0.790 & 0.771 & 0.756 & 0.743 & 0.732 & 0.723 \\
1.00 & 3.105 & 2.055 & 1.755 & 1.607 & 1.518 & 1.456 & 1.411 & 1.377 & 1.349 & 1.323 & 1.308 & 1.291 \\
\hline & & & & & & \multicolumn{10}{c}{ Sample size $n$} & & & & & \\
$\hat{C}_{\mathrm{pp}}$ & 70 & 80 & 90 & 100 & 110 & 120 & 130 & 140 & 150 & 160 & 170 & 180 \\
\hline 0.25 & 0.316 & 0.311 & 0.307 & 0.304 & 0.301 & 0.298 & 0.296 & 0.294 & 0.292 & 0.291 & 0.289 & 0.288 \\
0.36 & 0.455 & 0.448 & 0.442 & 0.437 & 0.433 & 0.429 & 0.426 & 0.423 & 0.421 & 0.419 & 0.417 & 0.415 \\
0.44 & 0.557 & 0.548 & 0.540 & 0.534 & 0.529 & 0.525 & 0.521 & 0.518 & 0.515 & 0.512 & 0.509 & 0.507 \\
0.56 & 0.708 & 0.697 & 0.688 & 0.680 & 0.673 & 0.668 & 0.669 & 0.659 & 0.655 & 0.651 & 0.648 & 0.646 \\
1.00 & 1.265 & 1.245 & 1.228 & 1.214 & 1.203 & 1.193 & 1.184 & 1.176 & 1.169 & 1.163 & 1.158 & 1.153 \\
\hline
\end{tabular}

(b)

\begin{tabular}{|c|c|c|c|c|c|c|c|c|c|c|c|c|}
\hline \multirow[b]{2}{*}{$C_{0}$} & \multicolumn{12}{|c|}{ Sample size $n$} \\
\hline & 5 & 10 & 15 & 20 & 25 & 30 & 35 & 40 & 45 & 50 & 55 & 60 \\
\hline 0.25 & 0.081 & 0.122 & 0.142 & 0.156 & 0.165 & 0.172 & 0.177 & 0.182 & 0.185 & 0.188 & 0.191 & 0.194 \\
\hline 0.36 & 0.116 & 0.175 & 0.205 & 0.224 & 0.237 & 0.247 & 0.255 & 0.261 & 0.267 & 0.271 & 0.275 & 0.279 \\
\hline 0.44 & 0.142 & 0.214 & 0.251 & 0.274 & 0.290 & 0.302 & 0.312 & 0.320 & 0.326 & 0.332 & 0.336 & 0.341 \\
\hline 0.56 & 0.180 & 0.272 & 0.319 & 0.348 & 0.369 & 0.385 & 0.397 & 0.407 & 0.415 & 0.422 & 0.428 & 0.434 \\
\hline \multirow[t]{2}{*}{1.00} & 0.322 & 0.487 & 0.570 & 0.622 & 0.659 & 0.687 & 0.708 & 0.726 & 0.741 & 0.754 & 0.765 & 0.774 \\
\hline & \multicolumn{12}{|c|}{ Sample size $n$} \\
\hline$C_{0}$ & 70 & 80 & 90 & 100 & 110 & 120 & 130 & 140 & 150 & 160 & 170 & 180 \\
\hline 0.25 & 0.198 & 0.201 & 0.204 & 0.206 & 0.208 & 0.210 & 0.211 & 0.213 & 0.214 & 0.215 & 0.216 & 0.217 \\
\hline 0.36 & 0.285 & 0.289 & 0.293 & 0.296 & 0.299 & 0.302 & 0.304 & 0.306 & 0.308 & 0.309 & 0.311 & 0.312 \\
\hline 0.44 & 0.348 & 0.354 & 0.358 & 0.362 & 0.366 & 0.369 & 0.372 & 0.374 & 0.376 & 0.378 & 0.380 & 0.382 \\
\hline 0.56 & 0.443 & 0.450 & 0.456 & 0.461 & 0.466 & 0.470 & 0.473 & 0.476 & 0.479 & 0.481 & 0.484 & 0.486 \\
\hline 1.00 & 0.790 & 0.803 & 0.814 & 0.824 & 0.832 & 0.839 & 0.845 & 0.850 & 0.855 & 0.860 & 0.864 & 0.868 \\
\hline
\end{tabular}

the factor $\chi_{n}^{2}(\alpha, \delta)\left(C_{0}-C_{\mathrm{ia}}\right) / n$ is the maximum value of the estimated incapability index $\hat{C}_{\mathrm{pp}}$ in order that the process is considered capable at least 100(1 $\alpha) \%$ of the time. Tables 5(b), 6(b), and 7(b) give the maximum values of $\hat{C}_{\mathrm{pp}}$ in the case with $\mu=T$ for the process to be considered capable (i.e., $C_{\mathrm{pp}} \leq C_{0}$ ) $90 \%, 95 \%$, and $99 \%$ of the time.

Suppose that the requirement for a process to be capable is that $C_{\mathrm{pp}} \leq 1.00$. We take a random sample of size $n$, and calculate $\hat{C}_{\mathrm{pp}}$. Using Table 6(b) based on the random sample of $n=30$, for example, we obtain $C_{0}=0.616$. Thus, if the calculated $\hat{C}_{\mathrm{pp}} \leq 0.616$, then we claim that the process is capable at least $95 \%$ of the time.

Copyright @ 2001 John Wiley \& Sons, Ltd.

\section{CONCLUSION}

Greenwich and Jahr-Schaffrath [1] introduced the process incapability index $C_{\mathrm{pp}}=C_{\mathrm{ip}}+C_{\mathrm{ia}}$, which provides an uncontaminated separation between information concerning the process precision $\left(C_{\mathrm{ip}}\right)$ and process accuracy $\left(C_{\mathrm{ia}}\right)$. In this note, we consider the three indices, and investigate the statistical properties of their natural estimators. For the three indices, we obtain their UMVUEs and MLEs. For each index, we compare the reliability of the two estimators based on their relative errors (square root of the relative mean squared error). In addition, we construct $90 \%, 95 \%$, and $99 \%$ upper confidence limits, 
Table 6. (a) The $95 \%$ upper confidence limits for $C_{\mathrm{pp}}$ under $\mu=T$, with given $\hat{C}_{\mathrm{pp}}$. (b) The maximum value of $\hat{C}_{\mathrm{pp}}$ under $\mu=T$ for which the process is capable $\left(C_{\mathrm{pp}} \leq C_{0}\right) 95 \%$ of the time

(a)

\begin{tabular}{ccccccccccccc}
\hline & \multicolumn{10}{c}{ Sample size $n$} \\
\cline { 2 - 13 }$\hat{C}_{\mathrm{pp}}$ & 5 & 10 & 15 & 20 & 25 & 30 & 35 & 40 & 45 & 50 & 55 & 60 \\
\hline 0.25 & 1.091 & 0.634 & 0.516 & 0.461 & 0.428 & 0.406 & 0.389 & 0.377 & 0.368 & 0.360 & 0.353 & 0.347 \\
0.36 & 1.571 & 0.914 & 0.744 & 0.664 & 0.616 & 0.584 & 0.561 & 0.543 & 0.529 & 0.518 & 0.508 & 0.500 \\
0.44 & 1.921 & 1.117 & 0.909 & 0.811 & 0.753 & 0.714 & 0.686 & 0.664 & 0.647 & 0.633 & 0.621 & 0.611 \\
0.56 & 2.444 & 1.421 & 1.157 & 1.032 & 0.958 & 0.908 & 0.872 & 0.845 & 0.823 & 0.805 & 0.791 & 0.778 \\
1.00 & 4.365 & 2.538 & 2.066 & 1.843 & 1.711 & 1.622 & 1.558 & 1.509 & 1.470 & 1.438 & 1.412 & 1.389 \\
\hline & & & & & & \multicolumn{10}{c}{ Sample size $n$} & & & & & \\
$\hat{C}_{\mathrm{pp}}$ & 70 & 80 & 90 & 100 & 110 & 120 & 130 & 140 & 150 & 160 & 170 & 180 \\
\hline 0.25 & 0.338 & 0.331 & 0.325 & 0.321 & 0.317 & 0.313 & 0.311 & 0.308 & 0.306 & 0.304 & 0.302 & 0.300 \\
0.36 & 0.487 & 0.477 & 0.469 & 0.462 & 0.456 & 0.451 & 0.447 & 0.443 & 0.440 & 0.437 & 0.435 & 0.432 \\
0.44 & 0.595 & 0.583 & 0.573 & 0.565 & 0.558 & 0.552 & 0.547 & 0.542 & 0.538 & 0.534 & 0.531 & 0.528 \\
0.56 & 0.758 & 0.742 & 0.729 & 0.719 & 0.710 & 0.702 & 0.696 & 0.690 & 0.685 & 0.680 & 0.676 & 0.672 \\
1.00 & 1.353 & 1.325 & 1.302 & 1.283 & 1.267 & 1.254 & 1.242 & 1.232 & 1.223 & 1.214 & 1.207 & 1.200 \\
\hline
\end{tabular}

(b)

\begin{tabular}{|c|c|c|c|c|c|c|c|c|c|c|c|c|}
\hline \multirow[b]{2}{*}{$C_{0}$} & \multicolumn{12}{|c|}{ Sample size $n$} \\
\hline & 5 & 10 & 15 & 20 & 25 & 30 & 35 & 40 & 45 & 50 & 55 & 60 \\
\hline 0.25 & 0.057 & 0.099 & 0.121 & 0.136 & 0.146 & 0.154 & 0.160 & 0.166 & 0.170 & 0.174 & 0.177 & 0.180 \\
\hline 0.36 & 0.082 & 0.142 & 0.174 & 0.195 & 0.210 & 0.222 & 0.231 & 0.239 & 0.245 & 0.250 & 0.255 & 0.259 \\
\hline 0.44 & 0.101 & 0.173 & 0.213 & 0.239 & 0.257 & 0.271 & 0.282 & 0.292 & 0.299 & 0.306 & 0.312 & 0.317 \\
\hline 0.56 & 0.128 & 0.221 & 0.271 & 0.304 & 0.327 & 0.345 & 0.359 & 0.371 & 0.381 & 0.389 & 0.397 & 0.403 \\
\hline \multirow[t]{2}{*}{1.00} & 0.229 & 0.394 & 0.484 & 0.543 & 0.584 & 0.616 & 0.642 & 0.663 & 0.680 & 0.695 & 0.708 & 0.720 \\
\hline & \multicolumn{12}{|c|}{ Sample size $n$} \\
\hline$C_{0}$ & 70 & 80 & 90 & 100 & 110 & 120 & 130 & 140 & 150 & 160 & 170 & 180 \\
\hline 0.25 & 0.185 & 0.189 & 0.192 & 0.195 & 0.197 & 0.199 & 0.201 & 0.203 & 0.204 & 0.206 & 0.207 & 0.208 \\
\hline 0.36 & 0.266 & 0.272 & 0.277 & 0.281 & 0.284 & 0.287 & 0.290 & 0.292 & 0.294 & 0.296 & 0.298 & 0.300 \\
\hline 0.44 & 0.325 & 0.332 & 0.338 & 0.343 & 0.347 & 0.351 & 0.354 & 0.357 & 0.360 & 0.362 & 0.365 & 0.367 \\
\hline 0.56 & 0.414 & 0.423 & 0.430 & 0.436 & 0.442 & 0.447 & 0.451 & 0.455 & 0.458 & 0.461 & 0.464 & 0.467 \\
\hline 1.00 & 0.739 & 0.755 & 0.768 & 0.779 & 0.789 & 0.798 & 0.805 & 0.812 & 0.818 & 0.823 & 0.829 & 0.833 \\
\hline
\end{tabular}

and the maximum values of $\hat{C}_{\mathrm{pp}}$ for which the process is capable. The results obtained in this paper are useful to the practitioners in choosing good estimators and making reliable decisions on judging process capability.

\section{APPENDIX}

Theorem 1. If the process characteristic is normally distributed, then:

(a) $\hat{C}_{\text {ip }}$ is the UMVUE of $C_{\mathrm{ip}}$;

(b) $\hat{C}_{\text {ip }}$ is consistent;

(c) $\sqrt{n}\left(\hat{C}_{\mathrm{ip}}-C_{\mathrm{ip}}\right)$ converges to $N\left(0,2 C_{\mathrm{ip}}^{2}\right)$ in distribution;

(d) $\hat{C}_{\text {ip }}$ is asymptotically efficient.

Copyright $\odot 2001$ John Wiley \& Sons, Ltd.
Proof. (a) Since $S_{n-1}^{2}$ is a sufficient and complete statistic for $\sigma^{2}$, and the unbiased estimator $\hat{C}_{\text {ip }}$ is a function $S_{n-1}^{2}$ of only, then by the Lehmann-Scheffe Theorem, $\hat{C}_{\text {ip }}$ is the UMVUE. (b) For all $\varepsilon>0$,

$$
\mathrm{P}\left\{\left|\hat{C}_{\text {ip }}-C_{\text {ip }}\right|>\varepsilon\right\}<\mathrm{E}\left(\hat{C}_{\text {ip }}-C_{\text {ip }}\right)^{2} / \varepsilon^{2}
$$

Since

$$
\mathrm{E}\left(\hat{C}_{\mathrm{ip}}-C_{\mathrm{ip}}\right)^{2}=\operatorname{Var}\left(\hat{C}_{\mathrm{ip}}\right)=2 C_{\mathrm{ip}}^{2} /(n-1)
$$

converges to zero, then $\hat{C}_{\text {ip }}$ must be consistent. (c) Greenwich and Jahr-Schaffrath [1] showed that, under general conditions, $\sqrt{n}\left(\hat{C}_{\text {ip }}-C_{\text {ip }}\right)$ converges to $N\left(0, \sigma_{\text {ip }}^{2}\right)$ in distribution, where $\sigma_{\mathrm{ip}}^{2}=\left(\mu_{4}-\right.$ $\left.\sigma^{4}\right) / D^{4}$. The result follows directly since for a normal 
Table 7. (a) The $99 \%$ upper confidence limits for $C_{\mathrm{pp}}$ under $\mu=T$, with given $\hat{C}_{\mathrm{pp}}$. (b) The maximum value of $\hat{C}_{\mathrm{pp}}$ under $\mu=T$ for which the process is capable $\left(C_{\mathrm{pp}} \leq C_{0}\right) 99 \%$ of the time

(a)

\begin{tabular}{|c|c|c|c|c|c|c|c|c|c|c|c|c|}
\hline \multirow[b]{2}{*}{$\hat{C}_{\mathrm{pp}}$} & \multicolumn{12}{|c|}{ Sample size $n$} \\
\hline & 5 & 10 & 15 & 20 & 25 & 30 & 35 & 40 & 45 & 50 & 55 & 60 \\
\hline 0.25 & 2.255 & 0.977 & 0.717 & 0.605 & 0.542 & 0.502 & 0.473 & 0.451 & 0.434 & 0.421 & 0.410 & 0.400 \\
\hline 0.36 & 3.247 & 1.407 & 1.033 & 0.872 & 0.781 & 0.722 & 0.681 & 0.650 & 0.625 & 0.606 & 0.590 & 0.576 \\
\hline 0.44 & 3.969 & 1.720 & 1.262 & 1.065 & 0.955 & 0.883 & 0.832 & 0.794 & 0.764 & 0.741 & 0.721 & 0.704 \\
\hline 0.56 & 5.051 & 2.189 & 1.606 & 1.356 & 1.215 & 1.123 & 1.059 & 1.011 & 0.973 & 0.943 & 0.917 & 0.896 \\
\hline \multirow[t]{2}{*}{1.00} & 9.020 & 3.909 & 2.868 & 2.421 & 2.169 & 2.006 & 1.891 & 1.805 & 1.737 & 1.683 & 1.638 & 1.601 \\
\hline & \multicolumn{12}{|c|}{ Sample size $n$} \\
\hline$\hat{C}_{\mathrm{pp}}$ & 70 & 80 & 90 & 100 & 110 & 120 & 130 & 140 & 150 & 160 & 170 & 180 \\
\hline 0.25 & 0.385 & 0.374 & 0.364 & 0.357 & 0.351 & 0.345 & 0.340 & 0.336 & 0.333 & 0.330 & 0.327 & 0.324 \\
\hline 0.36 & 0.555 & 0.538 & 0.525 & 0.514 & 0.505 & 0.497 & 0.490 & 0.484 & 0.479 & 0.475 & 0.471 & 0.467 \\
\hline 0.44 & 0.678 & 0.657 & 0.641 & 0.628 & 0.617 & 0.607 & 0.599 & 0.592 & 0.586 & 0.580 & 0.575 & 0.571 \\
\hline 0.56 & 0.863 & 0.837 & 0.816 & 0.799 & 0.785 & 0.773 & 0.763 & 0.754 & 0.746 & 0.738 & 0.732 & 0.726 \\
\hline 1.00 & 1.540 & 1.494 & 1.457 & 1.427 & 1.402 & 1.381 & 1.362 & 1.346 & 1.331 & 1.319 & 1.307 & 1.297 \\
\hline
\end{tabular}

(b)

\begin{tabular}{|c|c|c|c|c|c|c|c|c|c|c|c|c|}
\hline \multirow[b]{2}{*}{$C_{0}$} & \multicolumn{12}{|c|}{ Sample size $n$} \\
\hline & 5 & 10 & 15 & 20 & 25 & 30 & 35 & 40 & 45 & 50 & 55 & 60 \\
\hline 0.25 & 0.028 & 0.064 & 0.087 & 0.103 & 0.115 & 0.125 & 0.132 & 0.139 & 0.144 & 0.149 & 0.153 & 0.156 \\
\hline 0.36 & 0.040 & 0.092 & 0.126 & 0.149 & 0.166 & 0.179 & 0.190 & 0.199 & 0.207 & 0.214 & 0.220 & 0.225 \\
\hline 0.44 & 0.049 & 0.113 & 0.153 & 0.182 & 0.203 & 0.219 & 0.233 & 0.244 & 0.253 & 0.261 & 0.269 & 0.275 \\
\hline 0.56 & 0.062 & 0.143 & 0.195 & 0.231 & 0.258 & 0.279 & 0.296 & 0.310 & 0.322 & 0.333 & 0.342 & 0.350 \\
\hline \multirow[t]{2}{*}{1.00} & 0.111 & 0.256 & 0.349 & 0.413 & 0.461 & 0.498 & 0.529 & 0.554 & 0.576 & 0.594 & 0.610 & 0.625 \\
\hline & \multicolumn{12}{|c|}{ Sample size $n$} \\
\hline$C_{0}$ & 70 & 80 & 90 & 100 & 110 & 120 & 130 & 140 & 150 & 160 & 170 & 180 \\
\hline 0.25 & 0.162 & 0.167 & 0.172 & 0.175 & 0.178 & 0.181 & 0.184 & 0.186 & 0.188 & 0.190 & 0.191 & 0.193 \\
\hline 0.36 & 0.234 & 0.241 & 0.247 & 0.252 & 0.257 & 0.261 & 0.264 & 0.268 & 0.270 & 0.273 & 0.275 & 0.278 \\
\hline 0.44 & 0.286 & 0.294 & 0.302 & 0.308 & 0.314 & 0.319 & 0.323 & 0.327 & 0.330 & 0.334 & 0.337 & 0.339 \\
\hline 0.56 & 0.364 & 0.375 & 0.384 & 0.392 & 0.399 & 0.406 & 0.411 & 0.416 & 0.421 & 0.425 & 0.428 & 0.432 \\
\hline 1.00 & 0.649 & 0.669 & 0.686 & 0.701 & 0.713 & 0.724 & 0.734 & 0.743 & 0.751 & 0.758 & 0.765 & 0.771 \\
\hline
\end{tabular}

distribution, $\mu_{4}=3 \sigma^{4}$ and $C_{\text {ip }}=(\sigma / D)^{2}$. (d) Under the normality assumption, the information matrix can be calculated as follows. Since the information lower bound is achieved, then $\hat{C}_{\text {ip }}$ must be asymptotically efficient:

$$
\begin{gathered}
I(\theta)=I(\mu, \sigma)=\left[\begin{array}{cc}
1 / \sigma^{2} & 0 \\
0 & 1 /\left(2 \sigma^{4}\right)
\end{array}\right], \\
{\left[\frac{\partial C_{\mathrm{ip}}}{\partial \mu} \frac{\partial C_{\mathrm{ip}}}{\partial \sigma^{2}}\right] \frac{I^{-1}(\theta)}{n}\left[\begin{array}{c}
\frac{\partial C_{\mathrm{ip}}}{\partial \mu} \\
\frac{\partial C_{\mathrm{ip}}}{\partial \sigma^{2}}
\end{array}\right]=\frac{2 C_{\mathrm{ip}}^{2}}{n}}
\end{gathered}
$$

Theorem 2. If the process characteristic is normally distributed, then:

Copyright $\odot 2001$ John Wiley \& Sons, Ltd. (a) $\tilde{C}_{\mathrm{ia}}$ is the UMVUE of $C_{\mathrm{ia}}$;

(b) $\tilde{C}_{\text {ia }}$ is consistent;

(c) $\sqrt{n}\left(\tilde{C}_{\mathrm{ia}}-C_{\mathrm{ia}}\right)$ converges to $N\left(0,4 C_{\mathrm{ip}} C_{\mathrm{ia}}\right)$ in distribution;

(d) $\tilde{C}_{\mathrm{ia}}$ is asymptotically efficient.

Proof. (a) Noting that $\bar{X}$ is a sufficient and complete statistic for $\mu$, and that the unbiased estimator $\tilde{C}_{\mathrm{ia}}$ is a function of $\bar{X}$ only. By the Lehmann-Scheffe Theorem, $\tilde{C}_{\mathrm{ia}}$ is the UMVUE of $C_{\mathrm{ia}}$. (b) For any $\varepsilon>0$,

$$
\mathrm{P}\left\{\left|\tilde{C}_{\mathrm{ia}}-C_{\mathrm{ia}}\right|>\varepsilon\right\}<\mathrm{E}\left(\tilde{C}_{\mathrm{ia}}-C_{\mathrm{ia}}\right)^{2} / \varepsilon^{2}
$$

Since

$$
\mathrm{E}\left(\tilde{C}_{\mathrm{ia}}-C_{\mathrm{ia}}\right)^{2}=4 C_{\mathrm{ip}} C_{\mathrm{ia}} / n+\left[2 C_{\mathrm{ip}}^{2} /\left(n^{2}-n\right)\right]
$$

Qual. Reliab. Engng. Int. 2001; 17: 279-290 
converges to zero, then $\tilde{C}_{\text {ia }}$ must be consistent. (c) Greenwich and Jahr-Schaffrath [1] showed that under general conditions $\sqrt{n}\left(\hat{C}_{\mathrm{ia}}-C_{\mathrm{ia}}\right)$ converges to $N\left(0, \sigma_{\mathrm{ia}}^{2}\right)$ in distribution, where $\sigma_{\mathrm{ia}}^{2}=$ $4(\mu-T)^{2} \sigma^{2} / D^{4}$. Under the normality assumption, $\sqrt{n}\left(\hat{C}_{\mathrm{ia}}-C_{\mathrm{ia}}\right)$ must converge to $N\left(0,4 C_{\mathrm{ip}} C_{\mathrm{ia}}\right)$ in distribution. Since $\left(\tilde{C}_{\mathrm{ia}}-\hat{C}_{\mathrm{ia}}\right)$ converges to zero in probability, then by Slutsky's Theorem,

$$
\sqrt{n}\left(\tilde{C}_{\mathrm{ia}}-C_{\mathrm{ia}}\right)=\sqrt{n}\left(\tilde{C}_{\mathrm{ia}}-\hat{C}_{\mathrm{ia}}\right)+\sqrt{n}\left(\hat{C}_{\mathrm{ia}}-C_{\mathrm{ia}}\right)
$$

converges to $N\left(0,4 C_{\mathrm{ip}} C_{\mathrm{ia}}\right)$ in distribution. (d) Under the normality assumption, the information matrix can be calculated as follows. Since the information lower bound is achieved, then the estimator $\tilde{C}_{\mathrm{ia}}$ must be asymptotically efficient:

$$
\begin{gathered}
I(\theta)=I(\mu, \sigma)=\left[\begin{array}{cc}
1 / \sigma^{2} & 0 \\
0 & 1 /\left(2 \sigma^{4}\right)
\end{array}\right], \\
{\left[\frac{\partial C_{\mathrm{ia}}}{\partial \mu} \frac{\partial C_{\mathrm{ia}}}{\partial \sigma^{2}}\right] \frac{I^{-1}(\theta)}{n}\left[\begin{array}{c}
\frac{\partial C_{\mathrm{ia}}}{\partial \mu} \\
\frac{\partial C_{\mathrm{ia}}}{\partial \sigma^{2}}
\end{array}\right]=\frac{4 C_{\mathrm{ip}}^{2} C_{\mathrm{ia}}}{n}}
\end{gathered}
$$

Theorem 3. If the process characteristic is normally distributed, then:

(a) $\hat{C}_{\mathrm{pp}}$ is the MLE of $C_{\mathrm{pp}}$;

(b) $\hat{C}_{\mathrm{pp}}$ is the UMVUE of $C_{\mathrm{pp}}$;

(c) $\hat{C}_{\mathrm{pp}}$ is consistent;

(d) $\sqrt{n}\left(\hat{C}_{\mathrm{pp}}-C_{\mathrm{pp}}\right)$ converges to $N\left(0,2 C_{\mathrm{ip}} C_{\mathrm{ia}}+\right.$ $\left.2 C_{\mathrm{ip}} C_{\mathrm{pp}}\right)$ in distribution;

(e) $\hat{C}_{\mathrm{pp}}$ is asymptotically efficient.

Proof. (a) Since $\left(\bar{X}, S_{n}^{2}\right)$ is the MLE of $\left(\mu, \sigma^{2}\right)$, where $S_{n}^{2}=\sum_{i=1}^{n}\left(X_{i}-\bar{X}\right)^{2} / n$, and $\hat{C}_{\mathrm{pp}}=\left(S_{n}^{2} / D^{2}\right)+$ $\left[(\bar{X}-T)^{2} / D^{2}\right]$, then by the invariance property of the MLE, $\hat{C}_{\mathrm{pp}}$ is the MLE of $C_{\mathrm{pp}}$. (b) We note that $\left(\bar{X}, S_{n}^{2}\right)$ is sufficient and complete for $\left(\mu, \sigma^{2}\right)$. Since the unbiased estimator $\hat{C}_{\mathrm{pp}}$ is a function of $\left(\bar{X}, S_{n}^{2}\right)$ only, then by the Lehmann-Scheffe theorem $\hat{C}_{\mathrm{pp}}$ is the UMVUE. (c) For all $\varepsilon>0, \mathrm{P}\left\{\left|\hat{C}_{\mathrm{pp}}-C_{\mathrm{pp}}\right|>\varepsilon\right\}<$ $\mathrm{E}\left(\hat{C}_{\mathrm{pp}}-C_{\mathrm{pp}}\right)^{2} / \varepsilon^{2}$. Since $\mathrm{E}\left(\hat{C}_{\mathrm{pp}}-C_{\mathrm{pp}}\right)^{2}=\operatorname{Var}\left(\hat{C}_{\mathrm{pp}}\right)=$ $2 C_{\mathrm{ip}}\left(C_{\mathrm{ia}}+C_{\mathrm{pp}}\right) / n$ converges to zero, then $\hat{C}_{\mathrm{pp}}$ must be consistent. (d) Greenwich and Jahr-Schaffrath [1] showed that under general conditions $\sqrt{n}\left(\hat{C}_{\mathrm{pp}}-C_{\mathrm{pp}}\right)$ converges to $N\left(0, \sigma_{\mathrm{pp}}^{2}\right)$ in distribution, where

$$
\begin{aligned}
\sigma_{\mathrm{pp}}^{2}= & {\left[4(\mu-T)^{2} \sigma^{2} / D^{4}\right]+\left[4 \mu_{3}(\mu-T) / D^{4}\right] } \\
& +\left[\left(\mu_{4}-\sigma^{4}\right) / D^{4}\right]
\end{aligned}
$$

Therefore, $\quad \sqrt{n}\left(\hat{C}_{\mathrm{pp}}-C_{\mathrm{pp}}\right)$ converges to $N\left(0,2 C_{\mathrm{ip}} C_{\mathrm{ia}}+2 C_{\mathrm{ip}} C_{\mathrm{pp}}\right)$ in distribution if the process is normal. (e) Under the normality assumption, the information matrix can be calculated as follows. Since the information lower bound is achieved, then the estimator $\hat{C}_{\text {pp }}$ must be asymptotically efficient:

$$
\begin{gathered}
I(\theta)=I(\mu, \sigma)=\left[\begin{array}{cc}
1 / \sigma^{2} & 0 \\
0 & 1 /\left(2 \sigma^{4}\right)
\end{array}\right] \\
{\left[\frac{\partial C_{\mathrm{pp}}}{\partial \mu} \frac{\partial C_{\mathrm{pp}}}{\partial \sigma^{2}}\right] \frac{I^{-1}(\theta)}{n}\left[\begin{array}{c}
\frac{\partial C_{\mathrm{pp}}}{\partial \mu} \\
\frac{\partial C_{\mathrm{pp}}}{\partial \sigma^{2}}
\end{array}\right]=\frac{2 C_{\mathrm{ip}}}{n}\left(C_{\mathrm{ia}}+C_{\mathrm{pp}}\right)}
\end{gathered}
$$

\section{REFERENCES}

1. Greenwich M, Jahr-Schaffrath BL. A process incapability index. International Journal of Quality and Reliability Management 1995; 12(4):58-71.

2. Chan LK, Cheng SW, Spiring FA. A new measure of process capability: $C_{\mathrm{pm}}$. Journal of Quality Technology 1988; 20(3): 162-175.

3. Kane VE. Process capability indices. Journal of Quality Technology 1986; 18(1):41-52.

4. Pearn WL, Lin GH, Chen KS. Distributional and inferential properties of the process accuracy and process precision indices. Communications in Statistics: Theory and Methods 1998; 27(4):985-1000.

\section{Authors' biographies:}

W. L. Pearn is a Professor in the Department of Industrial Engineering and Management, National ChiaoTung University. He received his Ph.D. degree in operations research from the University of Maryland, College Park, MD, USA. He has published numerous papers in the areas of network optimization, scheduling, and process capability analysis.

G. H. Lin is a Lecturer in the Department of Telecommunications Engineering at the National Penghu Institute of Technology. He received his Ph.D. degree from the Department of Industrial Engineering and Management, National Chiao-Tung University. His research interests include applied statistics and quality management. 